\title{
A vendor-purchaser economic lot size problem with remanufacturing
}

DOI:

10.1007/s11573-014-0731-7

\section{Document Version}

Accepted author manuscript

Link to publication record in Manchester Research Explorer

\section{Citation for published version (APA):}

Pishchulov, G., Dobos, I., Gobsch, B., Pakhomova, N., \& Richter, K. (2014). A vendor-purchaser economic lot size problem with remanufacturing. Journal of Business Economics, 84(5), 749-791. https://doi.org/10.1007/s11573014-0731-7

\section{Published in:}

Journal of Business Economics

\section{Citing this paper}

Please note that where the full-text provided on Manchester Research Explorer is the Author Accepted Manuscript or Proof version this may differ from the final Published version. If citing, it is advised that you check and use the publisher's definitive version.

\section{General rights}

Copyright and moral rights for the publications made accessible in the Research Explorer are retained by the authors and/or other copyright owners and it is a condition of accessing publications that users recognise and abide by the legal requirements associated with these rights.

\section{Takedown policy}

If you believe that this document breaches copyright please refer to the University of Manchester's Takedown Procedures [http://man.ac.uk/04Y6Bo] or contact uml.scholarlycommunications@manchester.ac.uk providing relevant details, so we can investigate your claim.

\section{OPEN ACCESS}




\title{
A vendor-purchaser economic lot size problem with remanufacturing
}

\author{
Grigory Pishchulov, ${ }^{1}$ Imre Dobos, ${ }^{2}$ Barbara Gobsch, ${ }^{3}$ Nadezhda Pakhomova, ${ }^{4}$ Knut Richter ${ }^{4}$
}

${ }^{1}$ TU Dortmund University, Faculty of Business, Economics and Social Sciences, Martin-SchmeißerWeg 12, 44227 Dortmund, Germany

${ }^{2}$ Corvinus University of Budapest, Institute of Business Economics, Fövám tér 8, 1093 Budapest, Hungary

${ }^{3}$ European University Viadrina, Faculty of Business Administration and Economics, Große Scharrnstraße 59, 15230 Frankfurt (Oder), Germany

${ }^{4}$ Saint Petersburg State University, Faculty of Economics, 62 Tchaikovski street, 191123 St. Petersburg, Russia

grigory.pishchulov@tu-dortmund.de,imre.dobos@uni-corvinus.hu,bgobsch1@googlemail.com, n.pahomova@spbu.ru,k.rihter@spbu.ru

\begin{abstract}
In this paper, we study a closed-loop supply chain in which a single purchaser orders a particular product from a single vendor and sells it on the market. A certain fraction of used items return from the market back to the purchaser, who is responsible for collecting and returning them to the vendor. In addition to manufacturing new items, the vendor is able to remanufacture the returns into as-good-as-new items which are subsequently used to serve market demand. Our framework features the conventional joint economic lot size (JELS) model extended to include the return flow of the used items. In line with the assumptions of the JELS model, we assume a deterministic constant demand for the product. The fraction of used items returning from the market is assumed to depend on the purchaser's collection effort. To stimulate the returns, the vendor may offer the purchaser a transfer payment per item returned. The questions addressed by this study pertain to the optimal centralised control of this closed-loop supply chain, to the individually optimal policies of its members, and to the coordination within this supply chain under a decentralised control. In particular, we show that the transfer payment alone cannot coordinate the supply chain under consideration and may even fail to do so when combined with a two-part tariff - which is otherwise known to coordinate the corresponding forward supply chain. Our numerical study, though, has revealed that the combined contract is capable of substantially reducing the coordination deficit. We also introduce a novel three-part tariff which is shown to enable supply chain coordination in combination with the transfer payment.
\end{abstract}

Keywords: EOQ, joint economic lot size, remanufacturing, closed-loop supply chain, coordination

JEL Classification: C61, C71, M10

Published in Journal of Business Economics 84(5), 2014, pp. 749-791

The final publication is available at

http://link.springer.com/article/10.1007/s11573-014-0731-7 


\section{Introduction}

Collection of used products and their reuse has become in recent years the subject of increasing attention from both industrial practice and academic research due to important economic considerations, i.e., recovery of remaining product value, creation of an additional product value for the customers, protecting the market share from low-cost competitors, and maintaining the brand name by retaining control of the quality of remanufactured products (Corbett and Savaskan 2002, Atasu et al. 2010, Rubio et al. 2008). This "calls for a proper management of emerging closed-loop supply chains" (Dobos et al. 2013).

Academic research has addressed specific aspects of closed-loop supply chain management in a broad variety of settings, and this research can be divided into four streams (Atasu et al. 2008, see also Guide and Van Wassenhove 2009): (1) industrial engineering/ operations research approaches, which usually deal with the optimisation of specific activities; (2) work taking a more holistic view of product life cycle and supply-chain design and coordination; (3) work focusing on competition, market segmentation, and interaction between new and remanufactured products; and (4) studies addressing consumer choice and the effort of supply-chain members.

As in Dobos et al. (2013), we contribute to this growing body of research by studying a closed-loop supply-chain model that involves two independent firms entering a supplierbuyer relationship in the form of a bilateral monopoly, with both maintaining inventories of new and used products, which they control independently of each other and in their own interest. This focus makes our study most closely related to the first and second of the research streams mentioned above in that it deals with both operational and coordination aspects. More specifically, we study a closed-loop supply chain consisting of a vendor and a purchaser. The purchaser orders a single product from the manufacturer and sells it on the market. A certain fraction of used items return from the market back to the purchaser, who is responsible for collecting and returning them to the vendor. The vendor is able to remanufacture the returns into as-good-as-new items, which are subsequently used to serve market demand. The questions addressed by this study pertain, firstly, to the optimal centralised control of such a closed-loop supply chain; secondly, to the separate decisions of its members; and, thirdly, to their coordination.

While the first of the research streams has extensively investigated issues on the operational level, it typically does so by assuming a centralised control exerted on a production-inventory system by a single decision-maker. In contrast, the second of the research streams has investigated coordination among independent members of a closed-loop supply chain, but it usually adopts a marketing channel design perspective and lacks operational considerations. Therefore, the contribution of our work is threefold. Firstly, we study an optimal closed-loop supply-chain control according to a model that integrates both operational and tactical considerations. Secondly, we generalise a conventional model of bilateral monopoly to a closed-loop setting, thereby allowing us to compare between "traditional" (forward) and closed-loop supply-chain designs. Thirdly, we characterise solutions to all problems under study in the analytic form, thereby facilitating their evaluation and interpretation. We shall now relate our study to the existing literature in greater detail.

From the operational viewpoint, our study is most closely related to work that deals with inventory control and production planning in closed-loop settings. Akçalı and Çetinkaya (2011) have recently provided an overview of this body of literature and have classified it according to the assumptions of the individual studies with regard to: (a) the nature of demand and returns stream, (b) the number and function of stocking points, and (c) the form of control policy. However, Akçalı and Çetinkaya point to the fact that these studies usually assume a centralised control, and indicate that there is a need for research which deals with the issue of 
coordination between independent parties. Our work seeks to address this need. Of the studies surveyed by Akçalı and Çetinkaya (2011), the work most closely related to ours is that which assumes a single-product control over an infinite horizon with constant demand and return rates and perfect substitution between manufactured and remanufactured products (see Section 4.1 in Akçalı and Çetinkaya 2011 as well as Section 7.2 in Minner and Lindner 2004). The studies by Richter (1996a, b) in particular investigated a two-stage system in an EOQ-like setting with inventories of new and used products, and sought to determine an optimal centralised control policy in terms of lot size, number of manufacturing and remanufacturing batches per order cycle, and the product reuse rate, while assuming an infinite production rate for both manufacturing and remanufacturing (the latter assumption is also shared by other researchers; see, for example, Schrady 1967, Teunter 2001). The setting studied by Richter (1996a, b) represents in turn a generalisation of an earlier model (Richter 1994), one which assumes a control policy with at most one manufacturing and one remanufacturing batch per order cycle. We have therefore adopted this earlier model to represent the reverse channel in the closed-loop setting, as it directly matches the assumptions of the conventional joint economic lot size model of a forward supply chain, which serves as a reference model for our study (see below). In doing so, however, we also depart from the assumption of infinite production rates of manufacturing and remanufacturing that Richter (1994) made, and assume instead that both rates are finite, an assumption that is also made in much of the other work (Dobos and Richter 2004, Minner and Lindner 2004, Teunter 2004, Konstantaras and Skouri 2010, Jaber and El Saadany 2011). While these studies refer to more general control policies than we do, they still assume either a single-stage system or a fixed return rate of used products, or both. In contrast, our work considers a two-stage supply chain in which the return rate is subject to control and, as already mentioned, we also study coordination within this supply chain in a decentralised setting.

The problem of designing and coordinating a closed-loop supply chain has been addressed within the second of the research streams mentioned above. In particular, Savaskan et al. (2004) have studied several different reverse channel designs in a supply chain consisting of a manufacturer, a retailer, and optionally a third party to collect used products. Collecting the product requires a costly effort which ultimately determines the fraction of items returning from the market back to the manufacturer, who then remanufactures them at an equal cost to meet market demand in the next period. Savaskan et al. (2004) have found that delegating product collection to the retailer is most beneficial from the perspective of both individual members and society, since it results in higher profits for manufacturer and retailer, a lower sales price, and a higher rate of product return. Savaskan et al. also show that supply-chain coordination can be achieved with a two-part tariff in place of the wholesale price. This analysis has also been extended to the case of two competing retailers by Savaskan and Van Wassenhove (2006), to more general cost structures by Atasu et al. (2013), and to dualchannel collection by Hong et al. (2013).

However, this coordination analysis adopts a marketing channel design perspective, and lacks operational or inventory cost considerations, which may however be of importance (cf. Reyniers 2001). Subsequent work has addressed similar setups in non-cooperative settings (Zhao and Zhao 2011, Ma and Sun 2012, Wei et al. 2012, Nie et al. 2013) as well as different forms of contracting to achieve coordination (e.g. Shi et al. 2010, Cai 2011, Jun and Susheng 2011, Yan and Sun 2012, Govindan and Popiuc 2013). However, the same criticism can be levelled at this body of research, too. Our work differs in that we include operational and inventory-related considerations in our analysis while retaining the optimal channel design as established by Savaskan et al. (2004). We also adopt their assumptions regarding the form of relationship between supply-chain members as well as the nature of product returns. 
Furthermore, in line with Richter (1994) and Savaskan et al. (2004), we assume that the manufactured and remanufactured products are perfect substitutes.

To carry out this analysis, we refer to a conventional model that addresses both operational and coordination aspects in the context of a "traditional" (forward) supply chain. This model has received substantial attention in the literature, and assumes a number of different forms. In particular, Monahan (1984) has referred to a setting with a single purchaser that meets demand for a single product occurring at a constant rate, while ordering the product from a vendor who operates in the lot-for-lot fashion. In this setting, the vendor's quantity discounts were used as the means of coordination. Subsequently, Banerjee (1986a, b) has put forward a more general model that assumes a finite production rate at the vendor, and coined the term joint economic lot size (JELS). This work triggered broad interest in this and related supply-chain coordination problems, e.g., work addressing integrated production and shipment policies (see Ben-Daya et al. 2008 for a review), the coordination capability of various quantity discount forms (Kohli and Park 1989), product quality and setup cost reduction (Affisco et al. 2002, Liu and Çetinkaya 2007), and asymmetric information (Sucky 2006).

Like much of this work, we refer to Banerjee's JELS model since it is able to capture operations of supply-chain members, indicate the nature of conflict of interests between them, identify the means of coordination, and obtain closed-form solutions to the problem at hand (cf. Pibernik et al. 2011, Reyniers 2001). We contribute to this line of research by extending the conventional JELS model to a closed-loop setting, which, in addition to the coordination of lot-sizing between supply-chain members, also requires coordination with regard to the collection of used items. In line with Banerjee (1986a, b) and Savaskan et al. (2004), we assume symmetric information in this supply chain. Of those economic considerations indicated at the beginning of this section, we only focus on the first, i.e., on the opportunity for the manufacturer to recover the remaining value from used products; if the unit remanufacturing cost is lower than it is of manufacturing, then this represents an incentive for the manufacturer to engage in the collection of used products. As we will see in our analysis, this incentive can be further strengthened or weakened by the production rate of remanufacturing, and is diminished by the need to hold used products in stock.

The recent work by Liu et al. (2009) addresses a coordination problem similar to ours, one featuring an extension of the JELS model to a closed-loop supply chain. However, in their work, they do not capture the optimal policies analytically and generate insights entirely based on the simulation and numerical solution of the underlying optimization problems (see also Jonrinaldi and Zhang 2013). Our work differs in this respect as we pursue an analytic approach and obtain closed-form solutions which determine the structure of the optimal policies, thereby facilitating their evaluation and interpretation. However, for reasons of analytical tractability and to comply with the assumptions of the conventional JELS model, we restrict our analysis here to the case of a deterministic constant demand. To extend it to the case of a price-sensitive demand, as in Savaskan et al. (2004) and Liu et al. (2009), remains a future research opportunity.

We have addressed a number of problems that are closely related to the one studied here in other work. Dobos et al. (2011b) determine individually and jointly optimal remanufacturing and ordering policies in a simplified setting, for which Pishchulov et al. (2012) develop closed-form solutions and establish coordination deficit under two-part tariffs. Dobos et al. (2013) investigate equilibrium strategies of supply-chain members in a strategic game, and Dobos et al. (2011a) extend the basic model of Dobos et al. (2011b) to include unit (re-)manufacturing costs and a transfer payment for the returned products. Here, we further extend the latter study by addressing the collection of the product from the market and 
studying supply-chain coordination with bargaining on the transfer payment, the two-part tariff, and a novel three-part tariff.

Our paper is organised in the following way. Section 2 describes modelling assumptions and introduces the closed-loop supply-chain model underlying the study. Sections 3 and 4 characterise the optimal supply-chain control from the perspective of individual members and for the vertically integrated supply chain. Section 5 addresses the problem of coordinating the disintegrated supply chain. Section 6 concludes the paper with a summary of its main results and limitations and an outlook.

\section{The model}

\subsection{General description}

Consider a single purchaser facing a market demand for a homogeneous product at a constant rate of $D$ units per time unit. The purchaser serves the market demand from the inventory which is replenished repetitively by placing orders of the size $q$ with a single vendor who fills the orders in a lot-for-lot fashion. Note that an order of the size $q$ covers the purchaser's demand for the product over a time span of $T=q / D$ time units, which therefore represents the length of an order cycle at the purchaser. Furthermore, a fraction $\beta$ of the items sold return constantly over time from the market to the purchaser after a period of use. Following Savaskan et al. (2004), we assume $\beta$ to be a deterministic function of the purchaser's collection effort $I$, i.e., his investment in the promotion of product returns per time unit; we therefore call $\beta$ the collection rate. For reasons of analytical tractability, we also make the simplifying assumption that there is a linear dependence between $I$ and $\beta$ - expressed as $\beta=I / k$, where $k$ is a positive scaling constant. ${ }^{1}$ Furthermore, the purchaser incurs a collection and handling cost $a$ per unit returned, which is assumed to be exogenous to the model (see Liu et al. (2009) for an endogenous choice of this amount that in turn determines the collection rate). The used items collected by the purchaser are backhauled to the vendor by the same vehicle which delivers the purchaser's order. The vendor refunds the purchaser an amount $d$ - called the deposit - for each unit returned. By remanufacturing the used items returned - called nonserviceables - the vendor is able to produce as-good-as-new items, in addition to manufacturing new ones. Both kinds of items - called serviceables serve the purchaser's demand and, ultimately, that of the market. All nonserviceables are remanufactured, and therefore the collection rate $\beta$ represents at the same time the fraction of remanufactured items in the vendor's output and is called its remanufacturing rate. When in Section 5 we study the decentralised control in this closed-loop supply chain, we will assume in line with Savaskan et al. (2004) that the vendor has enough channel power to determine the deposit amount $d$ - which the purchaser then accepts and responds to with an order size $q$

and collection rate $\beta$ (the latter determined by the choice of collection effort $I$ ); furthermore, we will assume that the vendor has all the bargaining ability when it comes to bargaining with the purchaser. Figure 1 depicts the product flow in this supply chain as well as the parameters and decision variables employed in the model.

Inventories of serviceables and nonserviceables are held by both the vendor and the purchaser due to the constant cycle of production, delivery, collection and take-back of the product. The vendor's production rates $P_{M}, P_{R}$ of manufacturing and remanufacturing respectively, measured in units per time unit, are assumed to exceed the demand rate $D$ i.e., $P_{M}, P_{R}>D .^{2}$ The vendor and the purchaser incur fixed costs $s_{v}$ and $s_{p}$ per order 


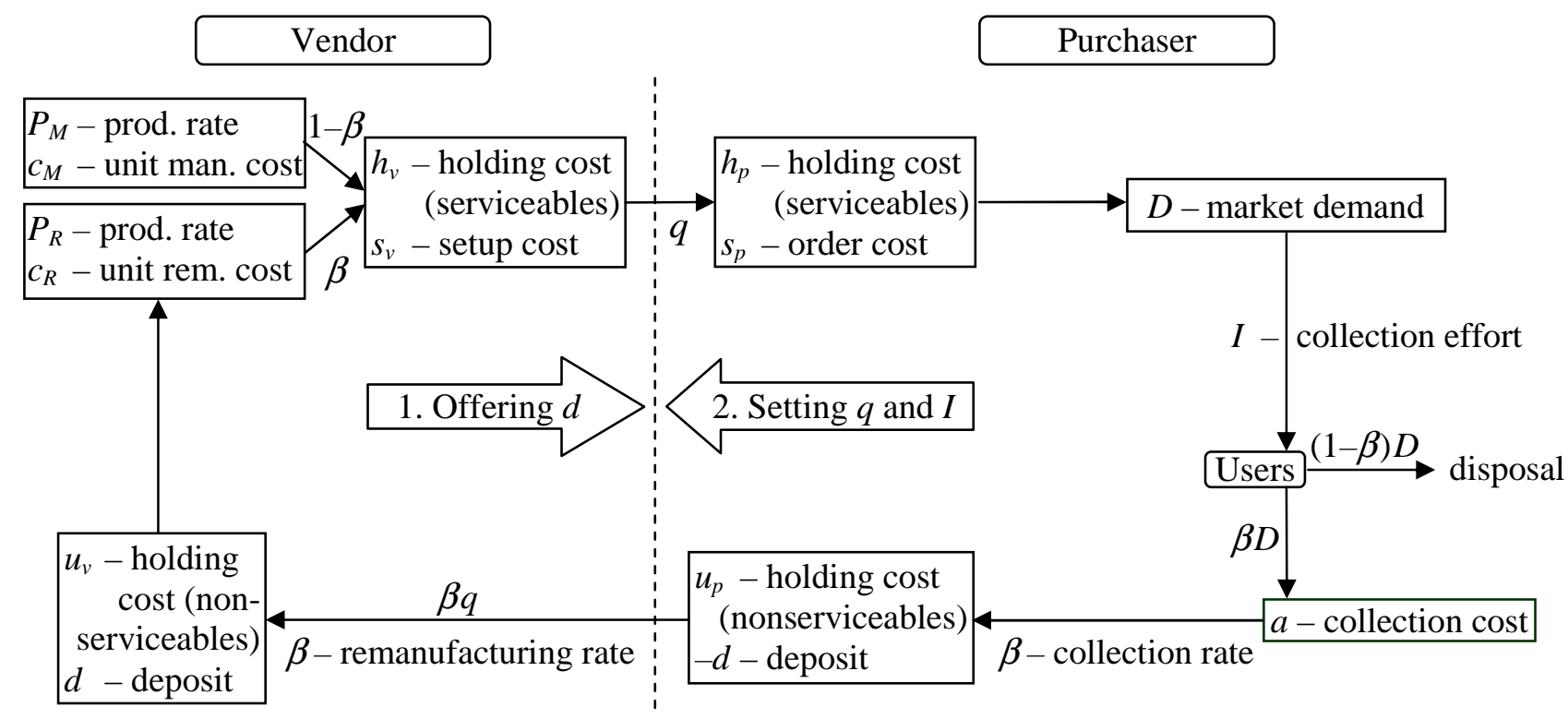

Fig. 1: Parameters and decision variables of the model

respectively, and inventory holding costs $h_{v}>u_{v}$ and $h_{p}>u_{p}$ per unit of serviceables and nonserviceables per time unit respectively. The unit manufacturing and remanufacturing costs are denoted by $c_{M}$ and $c_{R}$ respectively.

We will deal with the following complex of problems. The problems studied from the purchaser's perspective consist in determining the optimal order size for the given collection rate (problem PL) but also the optimal collection rate (problem PC). Regarding the vendor, we distinguish between two operational settings: one in which manufacturing takes place before remanufacturing (MbR), and the other which assumes the opposite (RbM). We will therefore determine the vendor's optimal lot size and the optimal remanufacturing rate in each of the two settings (problems VL-MbR, VC-MbR and VL-RbM, VC-RbM respectively). Addressing the centralised supply-chain control, we distinguish between the respective problems SL-MbR, SC-MbR, SL-RbM and SC-RbM. Finally, with regard to supply-chain coordination in the decentralised setting, we distinguish between VPC-MbR and VPC-RbM problems, and restrict our attention in this work to VPC-MbR and study this problem by considering three different contracts to be offered by the vendor to the purchaser, in the order of increasing complexity.

\subsection{Purchaser's cost function}

According to the above assumptions, the purchaser both depletes its inventory of serviceables and collects the nonserviceables at a constant rate, continuously over time. Consequently, its average inventory level of serviceables per order cycle is $q / 2$ and that of nonserviceables is $\beta q / 2$. The purchaser's total costs per time unit can therefore be expressed as

$$
T C_{p}(q, \beta)=C_{p}(q, \beta)+R_{p}(\beta)
$$

where

$$
C_{p}(q, \beta)=s_{p} \frac{D}{q}+\left(h_{p}+\beta u_{p}\right) \frac{q}{2} \quad \text { and } \quad R_{p}(\beta)=k \beta+(a-d) \beta D
$$




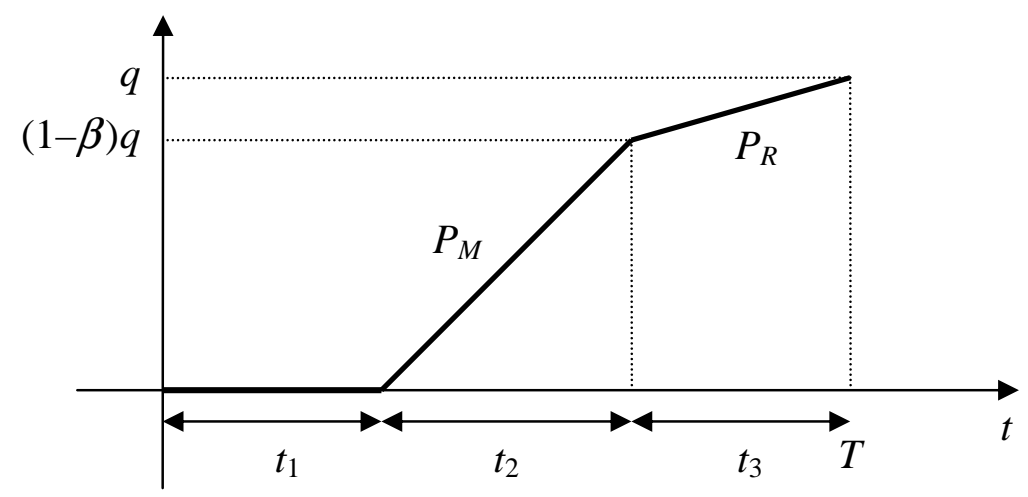

Fig. 2: The inventory level of serviceables at the vendor during an order cycle

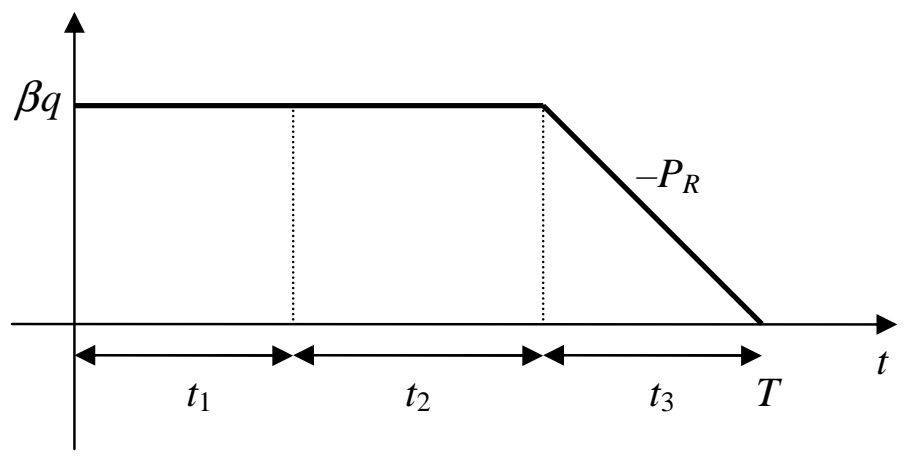

Fig. 3: The inventory level of nonserviceables at the vendor during an order cycle

represent the cost components which respectively do and do not depend on the order size. Note that the latter component, represented by the function $R_{p}(\beta)$, comprises the collection effort needed to maintain the return rate $\beta$, and costs of collecting and handling the volume $\beta D$ of returning items, less the deposit amount refunded by the vendor.

\subsection{Vendor's cost function: MbR setting}

The inventory levels of serviceables and nonserviceables at the vendor obviously depend on the choice of operational setting. Consider first the MbR setting which assumes that in each order cycle, $(1-\beta) q$ items are first manufactured and then $\beta q$ items are remanufactured. The corresponding analysis for the RbM setting and a comparison of both settings is presented in Section A.4.1 of the Appendix.

Figures 2 and 3 illustrate the evolution of the inventory level of serviceables and nonserviceables at the vendor during an order cycle, where $t_{2}$ and $t_{3}$ represent the duration of manufacturing and remanufacturing operations respectively, and $t_{1}=T-t_{2}-t_{3}$ is the slack time. Note that $t_{2}+t_{3}<T$ holds due to the assumption $P_{M}, P_{R}>D$ made in Section 2.1.

By virtue of Figures 2 and 3, the vendor's average stock-holding costs per time unit for the inventories of serviceables and nonserviceables respectively can be expressed as follows:

$$
H_{s}(q, \beta)=\frac{q}{2} h_{v} \cdot\left[(\beta-2) \beta \cdot\left(\frac{D}{P_{M}}-\frac{D}{P_{R}}\right)+\frac{D}{P_{M}}\right], \quad H_{n}(q, \beta)=\frac{q}{2} u_{v} \cdot\left(2 \beta-\frac{D}{P_{R}} \beta^{2}\right),
$$

which in total gives the following expression of the vendor's holding cost per time unit: 


$$
H_{v}(q, \beta)=\frac{q}{2} \cdot\left\{h_{v} \cdot\left[(\beta-2) \beta \cdot\left(\frac{D}{P_{M}}-\frac{D}{P_{R}}\right)+\frac{D}{P_{M}}\right]+u_{v} \cdot\left(2 \beta-\frac{D}{P_{R}} \beta^{2}\right)\right\} .
$$

Note that parameters $P_{M}$ and $P_{R}$ represent the speed at which the serviceables are produced by manufacturing and remanufacturing processes respectively, so that their magnitude determines the slopes of the respective line segments in Figures 2 and 3. The case of $P_{M}>P_{R}$ (depicted in Figure 2) therefore describes those situations where remanufacturing a used item requires more time than manufacturing a new one - if, for example, remanufacturing involves time-intensive disassembly, inspection, cleaning and reconditioning operations (see, for example, Steinhilper 1998, Matsumoto and Umeda 2011). Depending on the product, however, remanufacturing may happen to be the faster process as it eliminates the need to make certain components of the product from scratch (cf. Atasu and Çetinkaya 2006, Xu et al. 2012), which leads to the relation $P_{M}<P_{R}$. We might think here of refillable containers as an extreme example of such a product (cf. Atasu and Çetinkaya 2006). Note also that with $P_{M}=P_{R}$, the expression of the time-average costs $H_{s}(q, \beta)$ of holding the serviceable inventory at the vendor coincides with the respective cost expression in Banerjee's (1986a) joint economic lot size model. We refer the reader to Tang and Teunter (2006) for a case study with $P_{M} \approx P_{R}$.

By rearranging now the expression in the curly brackets in (4), we can identify the following three terms: one that does not depend on the remanufacturing rate $\beta$, one being linear in $\beta$, and one being quadratic in $\beta$, multiplied by the factors defined respectively as:

$$
V=h_{v} \frac{D}{P_{M}}, \quad \Omega_{M}=h_{v} \cdot\left(\frac{D}{P_{R}}-\frac{D}{P_{M}}\right)+u_{v} \quad \text { and } \quad \Delta_{M}=h_{v} \cdot\left(\frac{D}{P_{M}}-\frac{D}{P_{R}}\right)-u_{v} \frac{D}{P_{R}},
$$

so that

$$
H_{v}(q, \beta)=\frac{q}{2} \cdot\left\{\Delta_{M} \beta^{2}+2 \Omega_{M} \beta+V\right\} .
$$

It is easy to see that $V$ represents the rate at which an average unit in the production lot contributes to the vendor's holding costs if there is no remanufacturing $(\beta=0)$; if we refer to the partial derivative $\partial H_{v} / \partial \beta=q \cdot\left\{\Delta_{M} \beta+\Omega_{M}\right\}$, we can also observe that $\Omega_{M}$ represents the rate at which the holding costs per unit in the production lot will change when there is a marginal increase in collection rate; $\Delta_{M}$ therefore represents the rate of change of that rate with varying $\beta$. Note that the assumption $D<P_{R}$ made in Section 2.1 implies that $-\Omega_{M}<\Delta_{M}<V$. This in turn implies that $\Omega_{M}$ and $\Delta_{M}$ can never be both negative; further, with $\Omega_{M}>0$, it always holds that $\Delta_{M} \beta+\Omega_{M}>0$ for all $0 \leq \beta \leq 1$ - meaning that increasing $\beta$ under such circumstances will always increase the vendor's holding costs; with $\Omega_{M}<0$, $\Delta_{M} \beta+\Omega_{M}<0$ will hold for sufficiently small $\beta$ - meaning that remanufacturing can help reduce holding costs. We will discuss the nature and the meaning of parameters $\Delta_{M}, \Omega_{M}$ in Section 4.2 in greater detail. Finally, note that (6) is strictly positive for all $q>0$ and $0 \leq \beta \leq 1$ by construction of $H_{s}(q, \beta)$ and $H_{n}(q, \beta)$.

The vendor's ordering and holding costs per time unit can therefore be expressed as

$$
C_{v}(q, \beta)=s_{v} \frac{D}{q}+\frac{q}{2} \cdot\left\{\Delta_{M} \beta^{2}+2 \Omega_{M} \beta+V\right\} .
$$

The vendor also incurs costs which do not depend on the lot size, amounting per time unit to 


$$
R_{v}(\beta)=\left(d+c_{R}-c_{M}\right) \beta D+c_{M} D .
$$

Therefore, the vendor's total costs per time unit can be expressed as

$$
T C_{v}(q, \beta)=C_{v}(q, \beta)+R_{v}(\beta) .
$$

\subsection{The joint total costs}

Consider now the joint ordering and holding costs incurred by the parties per time unit:

$$
C_{T}(q, \beta)=\left(s_{v}+s_{p}\right) \frac{D}{q}+\frac{q}{2} \cdot\left\{\Delta_{M} \beta^{2}+\left(2 \Omega_{M}+u_{p}\right) \beta+V+h_{p}\right\}
$$

and let $W=V+h_{p}, U=2 \Omega_{M}+u_{p}$. Then (10) can be rewritten as

$$
C_{T}(q, \beta)=\left(s_{v}+s_{p}\right) \frac{D}{q}+\frac{q}{2} \cdot\left\{\Delta_{M} \beta^{2}+U \beta+W\right\}
$$

and the system-wide total costs per time unit expressed as

$$
T C_{T}(q, \beta)=C_{T}(q, \beta)+R_{T}(\beta)
$$

where $R_{T}(\beta)$ is the cost component that does not depend on the lot size, i.e.,

$$
R_{T}(\beta)=k \beta+\left(a+c_{R}-c_{M}\right) \beta D+c_{M} D .
$$

\section{Analysis of optimal lot sizes and minimum cost functions}

We first analyse the cost functions of the purchaser, the vendor and the whole system to determine the optimal order and lot sizes respectively.

The purchaser's PL problem is a variation of the classic economic order quantity (EOQ) model. The optimal order size for a given collection rate $\beta$ is then obviously expressed as

$$
q_{p}^{*}(\beta)=\sqrt{\frac{2 D s_{p}}{h_{p}+\beta u_{p}}}
$$

with the corresponding minimum total costs being

$$
T C_{p}^{*}(\beta)=\sqrt{2 D s_{p} \cdot\left(h_{p}+\beta u_{p}\right)}+R_{p}(\beta) .
$$

In the same way, the vendor's optimal lot size in the MbR setting (i.e., the optimal solution to the VL-MbR problem) is one that minimizes (9) and can be expressed as

$$
q_{v}^{*}(\beta)=\sqrt{\frac{2 D s_{v}}{\Delta_{M} \beta^{2}+2 \Omega_{M} \beta+V}} .
$$

The vendor's minimum total costs that thereby result can then be represented by the function

$$
T C_{v}^{*}(\beta)=\sqrt{2 D s_{v} \cdot\left(\Delta_{M} \beta^{2}+2 \Omega_{M} \beta+V\right)}+R_{v}(\beta) .
$$

It is easy to see that the sign of this function's second derivative does not depend on $\beta$; therefore, (17) is either convex or concave in $\beta$, or both. By analysing its first derivative and the function values at the boundary, we can therefore easily determine whether its minimum is attained at $\beta=0$, at $\beta=1$, or at some point in between. In the latter case, the optimal collection rate will be called non-trivial. In Section 4.2 below, we analyse this VC-MbR 


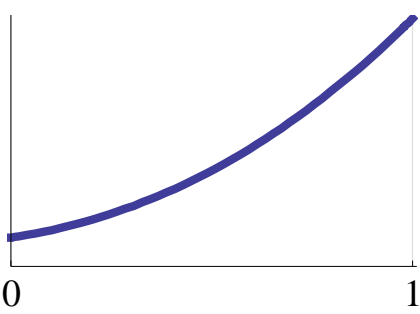

a

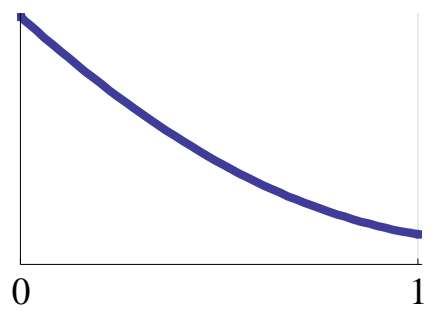

b

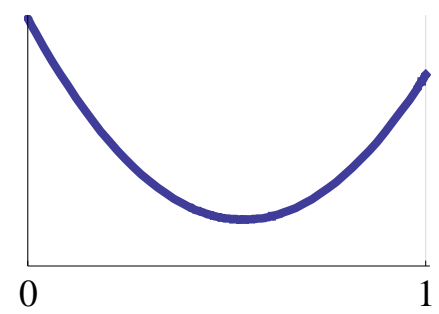

c

Fig. 4: Possible shapes of a strictly convex omnibus function $K(\beta)$

problem, while in Section A.4.2 in the Appendix we provide a solution to the VC-RbM problem.

Similarly, it follows from (10) to (12) that the system-wide optimal lot size (the solution to the SL-MbR problem) is given by

$$
q^{*}(\beta)=\sqrt{\frac{2 D \cdot\left(s_{v}+s_{p}\right)}{\Delta_{M} \beta^{2}+U \beta+W}}
$$

and implies the following minimum total costs system-wide:

$$
T C_{T}^{*}(\beta)=\sqrt{2 D \cdot\left(s_{v}+s_{p}\right)\left(\Delta_{M} \beta^{2}+U \beta+W\right)}+R_{T}(\beta) .
$$

Section A.4.3 in the Appendix provides the corresponding expression for the RbM setting.

\section{Optimal collection and remanufacturing rates}

Before we proceed with the analysis of the optimal collection and remanufacturing rates for the purchaser, the vendor and the whole system, it makes sense to utilise the fact that the expressions of the respective minimum cost functions (15), (17) and (19) are similarly structured, and to introduce the following "omnibus" function:

$$
K(\beta)=G \sqrt{A+B \beta^{2}+2 C \beta}+E \beta+F
$$

for which we also assume that

$$
A, G>0 \text { and } A+B \beta^{2}+2 C \beta>0 \text { for all } 0 \leq \beta \leq 1 .
$$

Obviously, $K(\beta)$ represents cost functions (15), (17) and (19) under a suitable definition of parameters $A$ to $C$ and $E$ to $G$. We study below basic properties of $K(\beta)$ to derive conclusions regarding optimal collection and remanufacturing rates from individual and joint perspectives.

We can work out quite easily that $K(\beta)$ is strictly convex if and only if $A B>C^{2}$, and is otherwise concave. If strictly convex, the function has a minimum in the interior of its domain whenever $K^{\prime}(0)<0$ and $K^{\prime}(1)>0-$ which, in turn, holds if and only if the inequalities

$$
\frac{C}{\sqrt{A}}<-\frac{E}{G}<\frac{B+C}{\sqrt{A+B+2 C}}
$$

hold. In all other cases, the minimum is found at one of the boundary points $\beta=0, \beta=1$. Note that inequality (22a) stands for the condition $K^{\prime}(0)<0-$ meaning essentially that increasing the collection rate from 0 up to a certain value would decrease the respective costs (see Figures $4 \mathrm{~b}, \mathrm{c}$ and $5 \mathrm{c}$ for an illustration). Similarly, inequality (22b) stands for the 
condition $K^{\prime}(1)>0$ - meaning that decreasing the collection rate from 1 to a lower value would decrease the costs (see Figures $4 \mathrm{a}, \mathrm{c}$ and $5 \mathrm{a}$ ). This provides us with the following:

Lemma 1. Let $A B>C^{2}$ hold. Then either (22a) or (22b) or both hold. Furthermore, $K(\beta)$ attains its minimum at

a) $\beta^{*}=0$ if (22a) does not hold while (22b) does,

b) $\beta^{*}=1$ if (22b) does not hold while (22a) does,

c) $\beta^{*}=-\frac{C}{B}-\frac{E}{B} \sqrt{\frac{A B-C^{2}}{B G^{2}-E^{2}}} \in(0,1)$

if $(22 a, b)$ both hold. The latter condition can only be fulfilled when $A B>C^{2}$.

Proof: See Appendix A.1.

Figures $4 \mathrm{a}$ to $4 \mathrm{c}$ illustrate the three possible shapes of a strictly convex function $K(\beta)$, as described in each case by a) to c) in Lemma 1 . Note that case c) leads to a non-trivial optimal collection/remanufacturing rate, determined by Lemma 1 in the analytical form. The following lemma treats the case of a concave $K(\beta)$.

Lemma 2. If $A B \leq C^{2}$, then $K(\beta)$ attains its minimum at $\beta^{*}=0$ whenever the inequality

$$
-\frac{E}{G}<\sqrt{A+B+2 C}-\sqrt{A}
$$

holds, and at $\beta^{*}=1$ whenever it holds in the opposite direction. The case of equality implies that both $\beta=0$ and $\beta=1$ deliver the minimum value to $K(\beta)$.

Proof: See Appendix A.2.

Figures 5a, b, 5c, d, and 5e each illustrate the three possible cases presented by Lemma 2.

We can now proceed to the analysis of the optimal collection rates for the purchaser, the vendor and the whole system. In this section, we again refer to the MbR setting only; the corresponding RbM analysis is conducted in Section A.4 in the Appendix.

\subsection{Purchaser's solution (problem PC)}

It is clear that the purchaser's minimum cost function (15) represents $K(\beta)$ with $A=h_{p}$, $B=0, C=u_{p} / 2, E=k+(a-d) D, F=0, G=\sqrt{2 D s_{p}}$, and complies with assumptions (21). Furthermore, it also complies with assumption $A B \leq C^{2}$ of Lemma 2, which therefore implies the following:

Proposition 1. The purchaser is not interested in collecting any used items $\left(\beta_{p}^{*}=0\right)$ if

$$
k+\left(\sqrt{h_{p}+u_{p}}-\sqrt{h_{p}}\right) \sqrt{2 D s_{p}}>(d-a) D .
$$

The purchaser will, however, commit to collecting them in their entirety $\left(\beta_{p}^{*}=1\right)$ if the above inequality holds in the opposite direction, and will be indifferent between no collection and full collection if the inequality becomes equality.

Hence, the purchaser's cost function is concave (cf. Figure 5), so that the purchaser always prefers a pure policy (i.e., no collection or full collection) and never a mixed policy (i.e., a non-trivial collection rate). The inequality in the condition of Proposition 1 can be 


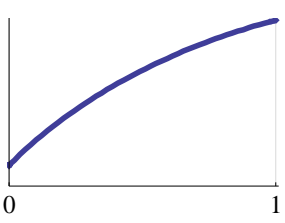

$\mathbf{a}$

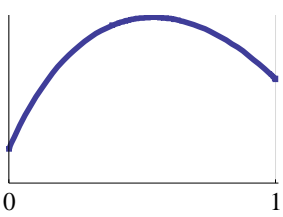

b

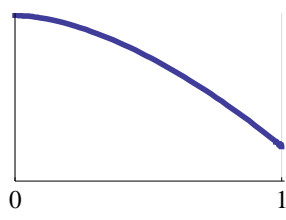

c

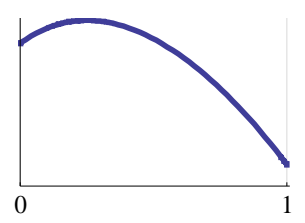

d

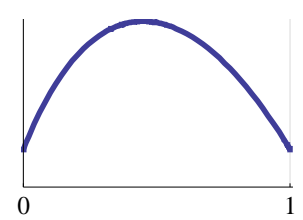

e

Fig. 5: Possible shapes of a concave omnibus function $K(\beta)$

immediately interpreted thus: if cost $k$ of promoting the returns and their unit holding cost $u_{p}$ are sufficiently high, while the net reward per returned item $(d-a)$ is sufficiently low, then the purchaser is not interested in the collection of used items. We can also refine this interpretation by rewriting the inequality in a straightforward way as follows:

$$
k+\left[\frac{1}{2} \mathbf{E} \mathbf{O Q} \mathbf{Q}_{1} \cdot\left(h_{p}+u_{p}\right)-\frac{1}{2} \mathbf{E} \mathbf{O Q} \mathbf{Q}_{\mathbf{0}} \cdot h_{p}\right] \cdot 2>(d-a) D,
$$

where $\mathbf{E} \mathbf{O Q} \mathbf{Q}_{\mathbf{0}}=q_{p}^{*}(0)$ is the purchaser's economic order quantity under conditions of no collection and $\mathbf{E} \mathbf{O Q} \mathbf{Q}_{1}=q_{p}^{*}(1)$, under conditions of full collection (see (14)). The bracketed expression therefore represents the increase in the holding costs per order cycle, should the purchaser switch from no collection to full collection. Furthermore, apart from increasing holding costs, full collection would also result in smaller order sizes, more order cycles per time unit, and, in effect, increased replenishment costs. Factor 2 after the brackets accounts for that increase, since we know for the economic order quantity that the total holding costs are equal to the total replenishment costs. Thus, the above inequality makes the following conclusion clear: if cost $k$ of promoting the full return of used products plus the increasing operational expenses (on the left) outweigh the total net reward per time unit (on the right), then the purchaser will not be committed to the collection of used products to any extent.

\subsection{Vendor's solution (VC-MbR)}

The vendor's minimum cost function (17) represents $K(\beta)$ with $A=V, B=\Delta_{M}, C=\Omega_{M}$, $E=\left(d+c_{R}-c_{M}\right) D, F=c_{M} D$ and $G=\sqrt{2 D s_{v}}$, and obviously complies with assumptions (21). By virtue of Lemmas 1 and 2, the following immediately holds:

Proposition 2. The vendor's optimal remanufacturing rate is non-trivial and is expressed as

$$
\beta_{v}^{*}=-\frac{\Omega_{M}}{\Delta_{M}}-\frac{E}{\Delta_{M}} \sqrt{\frac{V \Delta_{M}-\Omega_{M}^{2}}{2 D s_{v} \Delta_{M}-E^{2}}}
$$

whenever both of the following conditions hold:

$$
\frac{\Omega_{M}}{\sqrt{V}}<\frac{\left(c_{M}-c_{R}-d\right) D}{\sqrt{2 D s_{v}}}<\frac{\Omega_{M}+\Delta_{M}}{\sqrt{V+\Delta_{M}+2 \Omega_{M}}},
$$

and is otherwise $\beta_{v}^{*}=0$ or $\beta_{v}^{*}=1$.

Equation (27) is subject to the following interpretation. Assume for the moment that deposit $d$ equals the nominal saving $c_{M}-c_{R}$ made by the vendor to manufacturing costs through acquiring one unit of nonserviceables. In other words, we assume that the vendor's 
nominal benefit $c_{M}-c_{R}-d$ per unit of nonserviceables is zero. From the perspective of manufacturing costs alone, therefore, the vendor is indifferent towards the question of whether to acquire used products or not. However, as our discussion in Section 2.3 has revealed, increasing the remanufacturing rate $\beta$ can help the vendor to reduce its inventory holding costs, which applies to all such $\beta$ that $\Delta_{M} \beta+\Omega_{M}<0$ and all order sizes $q$. This is only possible if $\Omega_{M}<0$, since $-\Omega_{M}<\Delta_{M}$ holds by definition of the parameters in (5). Therefore, $\bar{\beta}=-\Omega_{M} / \Delta_{M}$ happens to be the non-trivial remanufacturing rate, up to which point the vendor's holding costs will decrease and beyond which, will increase. As this holds for any order size $q$, we therefore have the vendor's optimal remanufacturing rate $\beta_{v}^{*}=\bar{\beta}$ which yields (27), as $E=0$ due to the equality $d=c_{M}-c_{R}$. Note also that with $\Omega_{M}<0$, the inequality $\Omega_{M}+\Delta_{M}>0$ holds by definition, hence (28a, b) would both hold. This yields the following:

Corollary 1. If the vendor's nominal benefit $c_{M}-c_{R}-d$ per unit of nonserviceables is zero, then the optimal remanufacturing rate is non-trivial whenever $\Omega_{M}<0-$ which necessarily requires $P_{R}>P_{M}$, and zero otherwise.

Proof: See Appendix A.3.

Hence, if the vendor ever wishes to engage in remanufacturing in the MbR setting given the zero nominal benefit, then the vendor will always prefer a mixed policy; this is only possible if remanufacturing occurs at a faster pace than manufacturing. We can interpret Corollary 1 as follows. First, by definition of parameter $\Omega_{M}$, the inequality $\Omega_{M}<0$ translates to:

$$
u_{v}+h_{v} \cdot\left(\frac{D}{P_{R}}-\frac{D}{P_{M}}\right)<0 .
$$

If there is currently no remanufacturing $(\beta=0)$, then the first term above represents the contribution of one marginal unit of nonserviceables to the vendor's holding costs per time unit within an order cycle, should that unit be acquired (cf. Fig. 3). The second term then represents the net change in the holding costs of serviceables, should that unit be subsequently remanufactured (cf. Fig. 2). For the sake of simplicity, that marginal unit can be associated with a marginal increase of $\beta$. Note that, in the absence of remanufacturing $(\beta=0)$, the vendor produces and holds a stock of serviceables within the fraction $D / P_{M}$ of the entire order cycle. This implies that, over time, the vendor's holding cost per unit of serviceables per time unit will amount effectively to $h_{v} \cdot D / P_{M}$ (cf. Banerjee 1986a). Should remanufacturing completely supplant manufacturing $(\beta=1)$, the corresponding fraction of time would be $D / P_{R}$, and the corresponding effective holding cost $h_{v} \cdot D / P_{R}$. Consider now an increase in $\beta=0$ by a marginal amount $\delta$. In the context of the MbR setting, this implies that one last marginal unit of the production order is obtained by remanufacturing — after the rest of the production order has been manufactured. Then the net change in the production time of that last unit (taken relative to the length of the order cycle) amounts to $\left(D / P_{R}-D / P_{M}\right) \delta$. Thus, the fraction of time during which the newly manufactured items remain in stock also changes by the same amount, which triggers the change to the vendor's holding costs per unit of serviceables per time unit to the amount of $h_{v} \cdot\left(D / P_{R}-D / P_{M}\right) \delta$. Furthermore, before the last marginal unit is remanufactured, it must be kept throughout the order cycle in the nonserviceable inventory, which adds the amount $u_{v} \delta$ to the vendor's holding costs per unit 
of output per time unit. As a result, parameter $\Omega_{M}$ - expressed by the left-hand side in (29) - represents the sensitivity rate of the vendor's holding costs per unit of output per time unit with respect to a marginal increase of $\beta$; its negative value indicates a saving to the vendor's holding and total costs to be achieved if remanufacturing is introduced to a certain degree, and necessarily requires $P_{R}>P_{M}$ to hold. Note further that this sensitivity rate depends on the value of $\beta$, as Figures 2 and 3 suggest; its rate of change with varying $\beta$ is captured by the aggregate parameter $\Delta_{M}$, as defined in (5). Indeed, it can be seen in Figure 3 that, with increasing value of $\beta$, the increase in the costs of holding the nonserviceable inventory per time unit decreases at the rate $u_{v} \cdot D / P_{R}$. Figure 2 reveals in turn that, with increasing $\beta$, the rate of change in the effective costs of holding the serviceable inventory decreases in its magnitude, changing at the rate $h_{v} \cdot\left(D / P_{M}-D / P_{R}\right)$ due to the interplay of the following three effects: (i) remanufacturing affects the holding time of an ever smaller quantity of newly manufactured items, (ii) the time needed to manufacture them also becomes shorter, and (iii) an ever growing quantity of remanufactured items needs to be held in stock for a longer time until completion of the production order. This gives

$$
h_{v} \cdot\left(\frac{D}{P_{M}}-\frac{D}{P_{R}}\right)-u_{v} \frac{D}{P_{R}}
$$

as the rate at which the sensitivity of the vendor's holding costs per unit of output per time unit with respect to a marginal increase of $\beta$ changes with varying $\beta$, and represents parameter $\Delta_{M}$ as defined in (5). As a result, the sensitivity rate of the vendor's holding costs can be expressed for the given $\beta$ as $\Omega_{M}+\Delta_{M} \beta$. Note that inequality $\Omega_{M}<0$ (as expressed by (29)) implies $\Delta_{M}>0$, so that, in this case, the sensitivity rate initially decreases in its magnitude with increasing $\beta$ - until turning to zero, and then increases - to reach the value of $\Omega_{M}+\Delta_{M}>0$ at $\beta=1$. This holding cost behaviour can be explained as follows: the increase of $\beta$ gradually makes the reduction in the holding costs of serviceables vanish, whereas the rate of increase in the holding costs of nonserviceables, although decreasing, will never fully disappear (cf. Fig. 2 and 3) - so that, at some point, it becomes uneconomical to increase $\beta$ further - which leads to a non-trivial optimal remanufacturing rate. Obviously, with $\Omega_{M} \geq 0$, parameter $\Delta_{M}$ may or may not be negative; in either case, due to the inequality $\Omega_{M}+\Delta_{M}>0$, the said sensitivity rate $\Omega_{M}+\Delta_{M} \beta$ will never become negative - so that the holding costs will never decrease with increasing $\beta$ - which leads to the zero optimal remanufacturing rate.

A non-zero nominal benefit $c_{M}-c_{R}-d$ per unit of nonserviceables would take effect on the optimal remanufacturing rate in equation (27) via the aggregate parameter $E$. In particular, a positive nominal benefit implies a higher acceptance of remanufacturing by the vendor, yielding $E<0$ and hence an increase of $\beta_{v}^{*}$ in (27). Equations (A4) to (A8) in Appendix A.1 provide a further insight into the interplay between $\beta_{v}^{*}$ and the parameters under the radical in (27). Furthermore, it is worth making the following observation for the general case of an arbitrary nominal benefit. In Proposition 2, conditions (28a, b) together guarantee a non-trivial optimal remanufacturing rate. In assertion c) of Lemma 1, both conditions can simultaneously hold only if $A B>C^{2}$ holds - which by definition of parameters $A, B, C$ in the vendor's case immediately implies $\Delta_{M}>0$. By definition of $\Delta_{M}$ in (5), we therefore obtain the following: 
Corollary 2. For the vendor's optimal remanufacturing rate to be non-trivial, the following must necessarily hold:

$$
\frac{P_{R}}{P_{M}}>1+\frac{u_{v}}{h_{v}} .
$$

Hence, the requirement that remanufacturing occurs at a faster enough pace than manufacturing for the vendor to prefer a mixed policy holds for the MbR setting in general. This, however, does not necessarily prevent the vendor from engaging in remanufacturing, since a sufficiently high nominal benefit can encourage the vendor to adopt full remanufacturing, as the following discussion shows.

Indeed, it is interesting to provide the following interpretation for the willingness of the vendor to engage in remanufacturing in the MbR setting generally. Figures $4 \mathrm{~b}, \mathrm{c}$ and $5 \mathrm{c}$ illustrate the situations where the vendor's minimum total costs decrease immediately at $\beta=0$, leading to an optimal remanufacturing rate of $\beta_{v}^{*} \in(0,1]$. What these cases therefore have in common is that the vendor's minimum cost function (17) has a negative derivative at $\beta=0-$ which corresponds to condition (22a) or, equivalently, to (28a). We can rewrite the latter condition in the following way:

$$
\frac{\mathbf{E L S}_{\mathbf{0}}}{D} \cdot\left[h_{v} \cdot\left(\frac{D}{P_{R}}-\frac{D}{P_{M}}\right)+u_{v}\right]<c_{M}-c_{R}-d,
$$

where $\mathbf{E L S}_{0}=q_{v}^{*}(0)=\sqrt{2 P_{M} s_{v} / h_{v}}$ is the vendor's economic lot size in the absence of remanufacturing, as determined by (16) (see also Banerjee 1986a). The fraction $\mathbf{E L S}_{\mathbf{0}} / D$ to the left of the above inequality then represents the vendor's optimal order cycle length if there is currently no remanufacturing, while the bracketed expression represents the rate at which the vendor's holding costs per unit of output per time unit would change with a marginal increase of $\beta-$ as discussed above in this section. Hence, the left-hand side of the inequality represents the increase in the holding costs per order cycle in response to the remanufacturing of one marginal unit of nonserviceables, whereas the right-hand side represents the nominal benefit to be accrued by that. Hence, if the nominal benefit outweighs the increase in holding costs, then the vendor will be interested in engaging in remanufacturing.

It is however possible that the vendor's minimum cost function will not decrease immediately at $\beta=0$, but will do so at a later point — as illustrated in Figures $5 \mathrm{~b}$, d, e. This situation can only occur with a concave cost function - which obeys the assumption of Lemma 2 and necessarily requires $\Delta_{M}<0$ to hold. Accordingly, $\beta_{v}^{*} \in\{0,1\}$ and can be determined by verifying the function values at the boundary points. Figures $5 \mathrm{~d}$, e illustrate the case of $\beta_{v}^{*}=1$; for this to happen, the following condition due to Lemma 2 has to be satisfied:

$$
\left(\sqrt{V+\Delta_{M}+2 \Omega_{M}}-\sqrt{V}\right) \sqrt{2 D s_{p}} \leq\left(c_{M}-c_{R}-d\right) D .
$$

We can rewrite this in the following form:

$$
\left[\frac{1}{2} \mathbf{E L S}_{\mathbf{1}} \cdot \tilde{h}_{v}-\frac{1}{2} \mathbf{E L S}_{\mathbf{0}} \cdot h_{v} \frac{D}{P_{M}}\right] \cdot 2 \leq\left(c_{M}-c_{R}-d\right) D
$$

where $\mathbf{E L S}_{\mathbf{0}}$ is as defined above, $\mathbf{E L S}_{1}=q_{v}^{*}(1)=\sqrt{2 D s_{v} / \tilde{h}_{v}}$ is the vendor's economic lot size under the conditions of full collection as per (16), and $\tilde{h}_{v}=\Delta_{M}+2 \Omega_{M}+V=\left(h_{v}-u_{v}\right) \frac{D}{P_{R}}+2 u_{v}$ represents inventory holding costs accruing under the full collection policy per unit of product per time unit at the vendor. The above inequality is then subject to the same interpretation as 
the one provided for inequality (26) in Section 4.1 regarding the purchaser's optimal collection rate.

\subsection{The total system solution (SC-MbR)}

The system-wide total cost function (19) represents $K(\beta)$ with $A=W=V+h_{p}, B=\Delta_{M}$, $C=U / 2=\Omega_{M}+u_{p} / 2, E=k+\left(a+c_{R}-c_{M}\right) D, F=c_{M} D$ and $G=\sqrt{2 D \cdot\left(s_{v}+s_{p}\right)}$, and clearly complies with assumptions (21). Then again, by virtue of Lemmas 1 and 2, there holds the following:

Proposition 3. The system-optimal collection rate is non-trivial and expressed as

$$
\beta_{T}^{*}=-\frac{\Omega_{M}+u_{p} / 2}{\Delta_{M}}-\frac{E}{\Delta_{M}} \sqrt{\frac{\left(V+h_{p}\right) \Delta_{M}-\left(\Omega_{M}+u_{p} / 2\right)^{2}}{2 D \cdot\left(s_{v}+s_{p}\right) \Delta_{M}-E^{2}}}
$$

whenever both of the following conditions hold:

$$
\frac{\Omega_{M}+u_{p} / 2}{\sqrt{V+h_{p}}}<\frac{\left(c_{M}-c_{R}-a\right) D-k}{\sqrt{2 D \cdot\left(s_{v}+s_{p}\right)}}<\frac{\Omega_{M}+\Delta_{M}+u_{p} / 2}{\sqrt{V+\Delta_{M}+2 \Omega_{M}+h_{p}+u_{p}}},
$$

and is otherwise $\beta_{T}^{*}=0$ or $\beta_{T}^{*}=1$.

Proposition 3 is subject to much the same interpretation as the one supplied for Proposition 2 in the previous section, and we therefore omit it here for reasons of space. It is important, though, to state explicitly the following two corollaries, both of which can be proven and interpreted in a similar way to Corollaries 1 and 2 in the previous section.

Corollary 3. If the supply chain's nominal benefit $c_{M}-c_{R}-a$ per unit of nonserviceables equals the promotional effort $k / D$ needed to stimulate the return of that unit from the market, then the optimal collection rate from the supply-chain perspective is non-trivial whenever $\Omega_{M}<-u_{p} / 2-$ which necessarily requires $P_{R}>P_{M}$, and zero otherwise.

As we can immediately see, $\Omega_{M}<-u_{p} / 2$ represents a stronger condition than $\Omega_{M}<0$ of Corollary 1 in the vendor's case. This can be explained by the inclusion of the purchaser's holding costs in the analysis. For the general case there holds:

Corollary 4. For the supply chain's optimal collection rate to be non-trivial, condition (31) must necessarily hold.

As in the previous section, we provide the following interpretation to explain the attractiveness of collecting and remanufacturing used products in the MbR setting from the supply-chain perspective. The following condition stemming from (36a) indicates that, if fulfilled, it makes sense for the supply chain to engage in partial or full collection and remanufacturing:

$$
\frac{\mathbf{J E L S}_{\mathbf{0}}}{D} \cdot\left[h_{v} \cdot\left(\frac{D}{P_{R}}-\frac{D}{P_{M}}\right)+u_{v}+u_{p} / 2\right]<c_{M}-c_{R}-a-k / D .
$$

In the above, $\mathbf{J E L S}_{\mathbf{0}}=q^{*}(0)$ represents the classic joint economic lot size in the absence of remanufacturing, as determined by (18) (see also Banerjee 1986a). Hence, the left-hand side of the inequality represents the increase in the holding costs per production cycle in response to collecting and remanufacturing one marginal unit of nonserviceables, whereas the right- 
hand side represents the nominal benefit to be accrued by that, less the cost of the effort needed to acquire that item.

If the above condition does not hold, then it still makes sense for the supply chain to engage in full collection and remanufacturing if the following condition holds:

$$
k+\left[\frac{1}{2} \mathbf{J E L S}_{\mathbf{1}} \cdot\left(\tilde{h}_{p}+\tilde{h}_{v}\right)-\frac{1}{2} \mathbf{J E L S}_{\mathbf{0}} \cdot\left(h_{p}+h_{v} \frac{D}{P_{M}}\right)\right] \cdot 2 \leq\left(c_{M}-c_{R}-a\right) D
$$

where $\tilde{h}_{p}=h_{p}+u_{p}$ and $\tilde{h}_{v}=\Delta_{M}+2 \Omega_{M}+V=\left(h_{v}-u_{v}\right) \frac{D}{P_{R}}+2 u_{v}$ represent inventory holding costs accruing under the full collection policy per unit of product per time unit at the purchaser and the vendor respectively; $\mathbf{J E L S}_{1}=q^{*}(1)$ is the joint economic lot size under full collection as per (18), and $\mathbf{J E L S}_{\mathbf{0}}$ is as defined above. The above inequality is subject to the same interpretation as the one provided for inequality (26) in Section 4.1 regarding the purchaser's optimal collection rate.

\section{Coordinating the purchaser: the VPC-MbR problem}

In this section, we deal with the supply-chain coordination problem, assuming that the purchaser and the vendor are not vertically integrated and therefore pursue their own cost minimisation. We consider three different contracts in the order of increasing complexity, and show that the first two are not able to coordinate the supply chain under study - in the sense that the supply chain under these contract forms may perform worse than it does under centralised control. We also derive analytically the form of the third - coordinating contract. For reasons of space, we refer in this paper to the MbR setting only - which, according to Proposition A.1 (see Appendix), is beneficial for the vendor and the supply chain if and only if condition (A12) holds, something that requires the production rate of remanufacturing to exceed to a sufficient degree that of manufacturing.

\subsection{Contracting on the deposit amount}

Following Savaskan et al. (2004), we assume that the vendor has enough channel power to determine the amount of deposit $d$, which the vendor announces to the purchaser. In response, the purchaser chooses an order size $q$ and a collection rate $\beta$ (the latter according to the choice of the collection effort $I$ ) (see Figure 1). This assumption is suitable in, for example, a pull-supply-chain situation in which the vendor has full expertise in designing, manufacturing and remanufacturing the product and can therefore dictate the deposit amount, while the purchaser has full expertise in distributing and collecting the product and can therefore dictate the order size and collection rate (compare this with the choice of retail price and collection rate by the retailer in Savaskan et al. 2004). Assuming that the purchaser acts rationally and chooses such $q$ and $\beta$ as minimize its own costs given the deposit amount announced by the vendor, we can deal with the following problem of the vendor: what is the vendor's optimal deposit amount?

The problem under consideration therefore represents a Stackelberg game, in which the vendor is the leader and the purchaser, the follower; in more general terms, it represents a two-player extensive game with perfect information. The extensive game tree of that game is shown in Figure 6, where the players' payoffs at a terminal node have to be evaluated by means of equations (9) and (1) respectively. Due to the sequential nature of extensive games, a player's pure strategy represents a rule that prescribes a specific action to that player whenever he or she were to make a move in the course of the game, given every possible development (history) of the game as known to the player up until that point. A randomised 


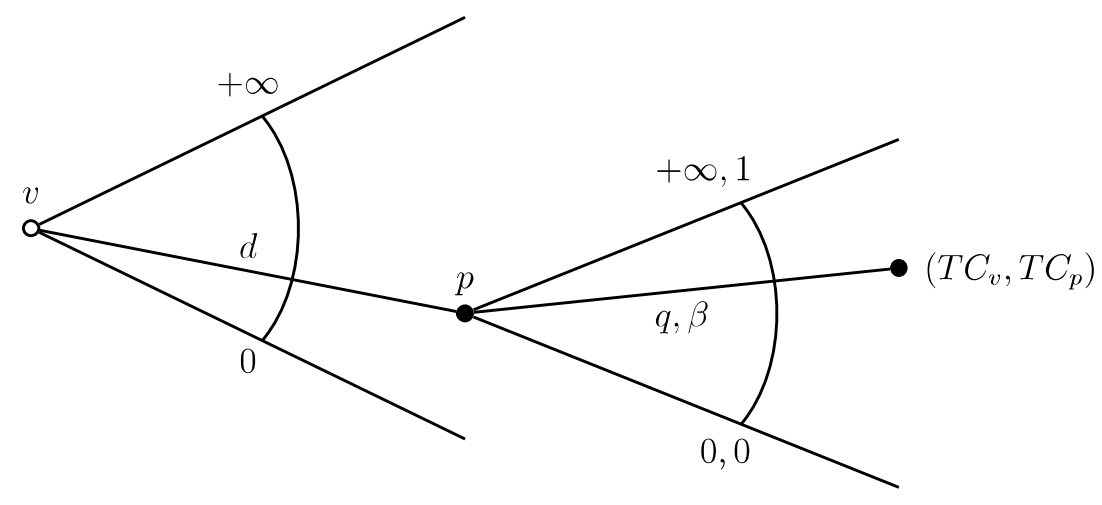

Fig. 6: Extensive game tree of the Stackelberg game ( $v$ and $p$ stand for vendor and purchaser, resp.)

choice of a pure strategy defines a mixed strategy. In a game with perfect information, the players' strategies comprise a subgame perfect equilibrium if, given every possible game history, none of the players can benefit from unilaterally deviating from his or her strategy. Note that in the extensive game under study, the vendor moves first by choosing a deposit amount $d$. Since the game history preceding this move is empty, and the vendor moves once only, we can equate the vendor's pure strategy $\sigma_{v}$ with the choice of the deposit amount: $\sigma_{v}=d$. The purchaser moves second by selecting an order size $q$ and a remanufacturing rate $\beta$ after observing the vendor's move. The purchaser is therefore perfectly informed with regard to the history of the game preceding his or her move, which enables the purchaser to understand perfectly the consequences of his or her own action. We can therefore represent a purchaser's strategy $\sigma_{p}$ after a history $d$ as $\sigma_{p}(d)=(q(d), \beta(d))$, and determine the subgame perfect equilibrium in pure strategies by means of backwards induction.

By taking into account the purchaser's optimal response, the vendor is able to foresee the consequences of his or her own choice of the deposit amount $d$ in terms of the total costs resulting - which we define as:

$$
\widetilde{T C}_{v}(d)=T C_{v}\left(q_{p}^{*}\left(\beta_{p}^{*}(d)\right), \beta_{p}^{*}(d)\right),
$$

where $T C_{v}(q, \beta)$ are the vendor's total costs as defined by $(9), \beta_{p}^{*}(d)$ is the purchaser's optimal collection rate as determined by Proposition 1 , and $q_{p}^{*}(\beta)$ is his or her optimal order size as defined by (14). Note that we have made $\beta_{p}^{*}(d)$ explicitly depend on $d$ in the notation; this reflects the fact that the purchaser's decision regarding the collection rate generally depends on the vendor's choice of the deposit amount. Also note that $q_{p}^{*}(\beta)$ does not explicitly depend on $d$; however, the purchaser's choice of the order size depends on $d$ via $\beta_{p}^{*}(d)$. Therefore, the vendor can use the deposit to influence (to a certain extent) the collection rate and the order size to be adopted within the supply chain. Note that according to Proposition 1, the purchaser's optimal collection rate is always a trivial one. Thus, the purchaser's equilibrium strategy can be expressed as:

$$
\sigma_{p}^{*}(d)= \begin{cases}\left(q_{p}^{*}(0), 0\right) & \text { for } d<\theta^{*} \\ \left(q_{p}^{*}(1), 1\right) & \text { for } d \geq \theta^{*}\end{cases}
$$

where 


$$
\theta^{*}=a+\frac{1}{D} \cdot\left[k+\left(\sqrt{h_{p}+u_{p}}-\sqrt{h_{p}}\right) \sqrt{2 D s_{p}}\right]>0
$$

represents the deposit threshold at which the purchaser's optimal strategy switches from no collection to full collection. We deliberately assume that $\beta_{p}\left(\theta^{*}\right)=1$, i.e., that the purchaser will choose an environmentally-friendly option when indifferent towards the two alternatives. Therefore, the purchaser's optimal order size can be either $q_{p}^{*}(0)$ (if $d<\theta^{*}$ ) or $q_{p}^{*}(1)$ (if $d \geq \theta^{*}$ ). Hence, (39) can be rewritten as

$$
\widetilde{T C}_{v}(d)=\left\{\begin{array}{ll}
s_{v} \frac{D}{q_{p}^{*}(0)}+\frac{q_{p}^{*}(0)}{2} h_{v} \frac{D}{P_{M}}+c_{M} D, & d<\theta^{*} \\
s_{v} \frac{D}{q_{p}^{*}(1)}+\frac{q_{p}^{*}(1)}{2}\left(\left(h_{v}-u_{v}\right) \frac{D}{P_{R}}+2 u_{v}\right)+\left(d+c_{R}\right) D, & d \geq \theta^{*}
\end{array} .\right.
$$

Thus, $\widetilde{T C}_{v}(d)$ is obviously constant for $d<\theta^{*}$ and increases in $d$ for $d \geq \theta^{*}$; hence, the vendor's choice of optimal deposit amount is either 0 or $\theta^{*}$ - whichever minimizes his or her total costs, and his equilibrium strategy is therefore:

$$
\sigma_{v}^{*}=\left\{\begin{array}{ll}
d_{v}^{*}=0, & \text { for } \widetilde{T C}_{v}(0)<\widetilde{T C}_{v}\left(\theta^{*}\right) \\
d_{v}^{*}=\theta^{*}, & \text { for } \widetilde{T C}_{v}(0) \geq \widetilde{T C}_{v}\left(\theta^{*}\right)
\end{array} .\right.
$$

In (43), we have deliberately assumed that, in the case of indifference towards the above two options, the vendor will choose the option that leads to an environmentally-friendly outcome. The above considerations directly yield the following result:

Proposition 4. The vendor's choice of deposit cannot lead to supply-chain coordination whenever a non-trivial collection rate is optimal for the supply chain under consideration.

Indeed, it is clear that the supply chain will lack coordination whenever the (uniquely defined) system-optimal collection rate is non-trivial - as it will not coincide with the purchaser's pure policy choice. Also, in those cases where the purchaser's collection rate turns out to be system-optimal, his or her choice of the order size does not necessarily coincide with the system-optimal one (cf. equations (14) and (18)). Therefore, the performance of the supply chain under the assumed decentralised control will not match its performance under the centralised control, leading to a coordination deficit.

Note also that Proposition 4 holds for the equilibrium strategy of the vendor as well as for any other deposit offer that the parties might attempt to adopt via a cooperative agreement. This leads us to analyse a bargaining problem in the next section.

\subsection{Contracting on the deposit amount and a two-part tariff}

It is known that introducing a suitable quantity discount (Cachon 2003) helps to achieve coordination in the forward supply chain - and in particular in the forward supply chain that complies with the assumptions of this work (Banerjee 1986a). We refer below to a quantitydiscount type of contract in the form of a two-part tariff (Dolan 1987) which is known to coordinate the corresponding forward supply chain (cf. Kohli and Park 1989). This is an "all unit" type of discount to be offered by the vendor to the purchaser, one which is specified by two entities: a fixed fee $w_{0}$ and a per-unit charge $w_{1}$, so that the amount payable is $w(q)=w_{0}+w_{1} q$, where $q$ is the order quantity. Thus, by adopting the order quantity $q$, the purchaser pays an amount of $\left(w_{0}+w_{1} q\right) \cdot D / q$ per time unit, where $D / q$ is the respective 
number of orders. The purchaser's cost function (1) is therefore adjusted to include the nowrelevant procurement costs:

$$
T C_{p}\left(d, q, \beta, w_{0}, w_{1}\right)=\left(s_{p}+w_{0}\right) \frac{D}{q}+\left(h_{p}+\beta u_{p}\right) \frac{q}{2}+R_{p}(d, \beta)+w_{1} D .
$$

We naturally assume that (44) is defined for all $w_{0}>-s_{p}$ and all $w_{1}$. Note that we have made $T C_{p}$ and $R_{p}$ explicitly depend on $d$ in the notation. It is then clear that the purchaser's minimum total costs (15) can now be expressed as follows:

$$
T C_{p}^{*}\left(d, \beta, w_{0}, w_{1}\right)=\sqrt{2 D \cdot\left(s_{p}+w_{0}\right)\left(h_{p}+\beta u_{p}\right)}+R_{p}(d, \beta)+w_{1} D .
$$

Obviously, the concave behaviour of the purchaser's minimum total costs as a function of $\beta$ remains, as does the choice of a pure collection policy by the purchaser for the given $d, w_{0}, w_{1}$, which can be expressed as

$$
\beta_{p}^{*}\left(d, w_{0}\right)= \begin{cases}0, & d<\theta^{*}\left(w_{0}\right) \\ 1, & d \geq \theta^{*}\left(w_{0}\right)\end{cases}
$$

with

$$
\theta^{*}\left(w_{0}\right)=a+\frac{1}{D} \cdot\left[k+\left(\sqrt{h_{p}+u_{p}}-\sqrt{h_{p}}\right) \sqrt{2 D \cdot\left(s_{p}+w_{0}\right)}\right]
$$

being the deposit threshold that applies regarding the same considerations as in Section 5.1 above. Hence, the introduction of the above two-part tariff does not help to coordinate the supply chain under consideration, either, whenever the system-optimal collection rate is nontrivial. It is, however, worthwhile to develop insights into the following question:

1) What choice of quantity discount parameters $w_{0}, w_{1}$ and deposit amount $d$ is most favourable to the vendor while still being acceptable to the purchaser?

2) To what extent does this type of contract help to reduce the coordination deficit of the deposit-based control analysed in Section 5.1, and what is the extent of the remaining coordination deficit?

We address the first question in the remainder of this section, and the second question in Section 5.3 below.

First, it is useful to gain the following insight into the effect that the quantity discount parameters $w_{0}, w_{1}$ and the deposit amount $d$ have on the optimal decisions of the purchaser. From the above definition of $\theta^{*}\left(w_{0}\right)$, the following obviously holds:

Proposition 5. $\theta^{*}\left(w_{0}\right)$ is positive and increases monotonically in $w_{0}$.

Indeed, with $u_{p}>0$ by assumption, the difference between two radicals in the definition of $\theta^{*}\left(w_{0}\right)$ is positive. Then, with $a, D, k>0$ by assumption, $\theta^{*}\left(w_{0}\right)$ is positive as well. Note further that $s_{p}>-w_{0}$ holds by assumption, and thus the last radical in the definition of $\theta^{*}\left(w_{0}\right)$ is well-defined. Taking into account that this radical is multiplied by a positive factor leads to the assertion of the proposition. Thus, a higher fixed fee $w_{0}$ would imply a higher deposit amount at which the purchaser's collection policy would switch from no collection to full collection. Indeed, the fixed fee $w_{0}$ effectively adds to the purchaser's fixed ordering costs, so that increasing $w_{0}$ increases the purchaser's optimal order size for the given $\beta$ and, ultimately, the purchaser's inventory-related costs (expressed by the radical in (45)). Since these costs increase by the same factor under no-collection and full-collection policies, so does the absolute difference between them. This requires, according to the same argument as 
made following Proposition 1 in Section 4.1, a stronger incentive for the purchaser in the form of a higher deposit refund for switching from no collection to full collection.

Further, if we use the same approach as in Section 3, then we can express the purchaser's optimal order size for the given collection rate $\beta$ and fixed fee $w_{0}$ as

$$
q_{p}^{*}\left(\beta, w_{0}\right)=\sqrt{\frac{2 D \cdot\left(s_{p}+w_{0}\right)}{h_{p}+\beta u_{p}}},
$$

being independent of the per-unit charge $w_{1}$. Then, according to Proposition 5 and the definition of the purchaser's optimal collection rate $\beta_{p}^{*}\left(d, w_{0}\right)$ in (46), the following holds:

Proposition 6. For any fixed deposit amount $d, \beta_{p}^{*}\left(d, w_{0}\right)$ does not increase in $w_{0}$, whereas $q_{p}^{*}\left(\beta_{p}^{*}\left(d, w_{0}\right), w_{0}\right)$ increases monotonically in $w_{0}$.

Indeed, by Proposition 5, increasing $w_{0}$ makes $\theta^{*}\left(w_{0}\right)$ increase. This, in turn, either retains $\beta_{p}^{*}\left(d, w_{0}\right)$ on the same level or makes it decrease from 1 (full collection) to 0 (no collection). Furthermore, equation (48) makes clear that $q_{p}^{*}\left(\beta, w_{0}\right)$ grows monotonically with $w_{0}$. Noting that collection rate $\beta$ appears in the denominator in (48) and knowing that $\beta_{p}^{*}\left(d, w_{0}\right)$ does not increase with $w_{0}$ leads to the conclusion that $q_{p}^{*}\left(\beta_{p}^{*}\left(d, w_{0}\right), w_{0}\right)$ increases with $w_{0}$. Thus, increasing the fixed fee $w_{0}$ while keeping the deposit amount $d$ fixed can only decrease the purchaser's optimal collection rate and will strictly increase his or her optimal order size. By a similar analysis, we can show that increasing $d$ while keeping $w_{0}$ fixed can only increase the purchaser's optimal collection rate and, as a consequence, only decrease his optimal order size. Note also that the per-unit charge $w_{1}$ does not influence the purchaser's optimal decision with regard to the collection rate and the order size.

Next, consider the effect that the two-part tariff $w_{0}, w_{1}$ has on the parties' individual costs. According to (45) and (46), the purchaser's minimum total costs for the given $d, w_{0}, w_{1}$ are:

$$
T C_{p}^{*}\left(d, w_{0}, w_{1}\right)=\left\{\begin{array}{ll}
\sqrt{2 D \cdot\left(s_{p}+w_{0}\right) h_{p}}+w_{1} D, & d<\theta^{*}\left(w_{0}\right) \\
\sqrt{2 D \cdot\left(s_{p}+w_{0}\right)\left(h_{p}+u_{p}\right)}+k+\left(a-d+w_{1}\right) D, & d \geq \theta^{*}\left(w_{0}\right)
\end{array} .\right.
$$

Adjusting in turn the vendor's total cost function for the revenue gained yields

$$
T C_{v}\left(d, q, \beta, w_{0}, w_{1}\right)=\left(s_{v}-w_{0}\right) \frac{D}{q}+\frac{q}{2} \cdot\left\{\Delta_{M} \beta^{2}+2 \Omega_{M} \beta+V\right\}+R_{v}(d, \beta)-w_{1} D
$$

(where dependence on the deposit is made explicit wherever it applies). Thus, the vendor's costs caused by the purchaser's optimal choice of a collection rate and an order size can be expressed as

$$
\widetilde{T C}_{v}\left(d, w_{0}, w_{1}\right)=T C_{v}\left(d, q_{p}^{*}\left(\beta_{p}^{*}\left(d, w_{0}\right), w_{0}\right), \beta_{p}^{*}\left(d, w_{0}\right), w_{0}, w_{1}\right) .
$$

Taking (46) and (48) into account, we can rewrite the above as

$$
\widetilde{T C}_{v}\left(d, w_{0}, w_{1}\right)= \begin{cases}\left(s_{v}-w_{0}\right) \frac{D}{q_{p}^{*}\left(0, w_{0}\right)}+\frac{q_{p}^{*}\left(0, w_{0}\right)}{2} h_{v} \frac{D}{P_{M}}+c_{M} D-w_{1} D, & d<\theta^{*}\left(w_{0}\right) \\ \left.\left(s_{v}-w_{0}\right) \frac{D}{q_{p}^{*}\left(1, w_{0}\right)}+\frac{q_{p}^{*}\left(1, w_{0}\right)}{2}\left(h_{v}-u_{v}\right) \frac{D}{P_{R}}+2 u_{v}\right)+\left(d+c_{R}\right) D-w_{1} D, & d \geq \theta^{*}\left(w_{0}\right)\end{cases}
$$


The first study question stated above leads us to consider a setting in which the parties agree to act cooperatively and determine the contract parameters $d, w_{0}, w_{1}$ via bargaining. In so doing, we assume in line with Savaskan et al. (2004) that the vendor has all the bargaining ability (the opposite case can be treated similarly). The bargaining problem that results from this represents a principal-agent problem with the vendor acting as the principal and the purchaser as the agent (Ross 1973, Myerson 1983). The problem at hand is also characterised by the property of complete information in the sense that the parties are fully informed about each other's problem-specific data, and have full certainty with regard to the state of the nature to occur - in the sense that the outcome of decisions made is not affected by random occurrences. The problem allows the following modelling and solution (cf. Banerjee 1986a, p. 304ff.). The vendor seeks to find such a deposit amount and a two-part tariff which would minimize the vendor's total costs; the vendor should, however, make an offer to the purchaser such that it does not increase the purchaser's total costs compared to the non-cooperative equilibrium without the two-part tariff (as characterised in Section 5.1 above) - since otherwise the purchaser will not be inclined to accept the contract. Thus, the vendor faces the following problem:

$$
\min _{d \geq 0} \widetilde{T C}_{v}\left(d, w_{0}, w_{1}\right) \quad \text { s.t. } \quad T C_{p}^{*}\left(d, \beta_{p}^{*}\left(d, w_{0}\right), w_{0}, w_{1}\right) \leq T C_{p}^{*}\left(d_{v}^{*}, \beta_{p}^{*}\left(d_{v}^{*}, 0\right), 0, \bar{w}\right)
$$

where $\bar{w}$ is the wholesale price prior to the introduction of the discount. Note that according to (43), the vendor's equilibrium deposit choice $d_{v}^{*}$ can be either 0 or $\theta^{*}(0)$. It is easy to verify, though, that either choice implies the same total costs for the purchaser:

$$
T C_{p}^{*}\left(0, \beta_{p}^{*}(0,0), 0, \bar{w}\right)=T C_{p}^{*}\left(\theta^{*}(0), \beta_{p}^{*}\left(\theta^{*}(0), 0\right), 0, \bar{w}\right)=\sqrt{2 D s_{p} h_{p}}+\bar{w} D .
$$

Taking (49) into account, we can rewrite the constraint in (53) as follows:

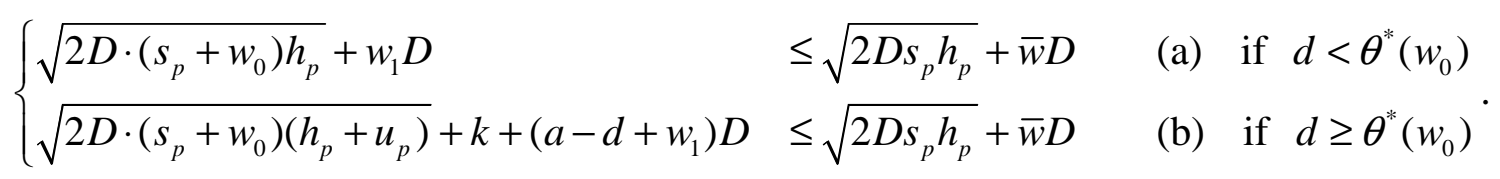

Note that in both $(55 \mathrm{a}, \mathrm{b})$, the right-hand side represents the purchaser's total costs in the noncooperative equilibrium (i.e., the purchaser's status quo), while the left-hand side represents the purchaser's total costs given the vendor's offer. In (55a), the vendor's offer will not lead to any collection because the deposit amount is insufficient, and this can therefore be set to $d=0$. In (55b), though, the deposit amount $d$ will lead to full collection. Note also that for any fixed $w_{0}, w_{1}$, the vendor's cost function (52) is obviously constant in $d$ for $d<\theta^{*}\left(w_{0}\right)$ and increases for $d \geq \theta^{*}\left(w_{0}\right)$. Hence, the vendor's problem (53) can be solved by separating it into the following two subproblems and comparing their optimal objective values:

$$
\begin{array}{ll}
\widetilde{T C}_{v}^{1}:=\min _{w_{0}, w_{1}} \widetilde{T C}_{v}\left(0, w_{0}, w_{1}\right) & \text { s.t. }(55 \mathrm{a}), \\
\widetilde{T C}_{v}^{2}:=\min _{w_{0}, w_{1}} \widetilde{T C}_{v}\left(\theta^{*}\left(w_{0}\right), w_{0}, w_{1}\right) \quad \text { s.t. }(55 \mathrm{~b}) .
\end{array}
$$

If we then let

$$
i^{*}=\underset{i \in\{1,2\}}{\arg \min } \widetilde{T C}_{v}^{i}
$$

then the vendor's optimal choice of the quantity discount can be expressed as

$$
\left(w_{0}^{*}, w_{1}^{*}\right)=\arg \left(56 . i^{*}\right)
$$

and that of the deposit amount as: 


$$
d^{*}=\left\{\begin{array}{ll}
0, & i^{*}=1 \\
\theta^{*}\left(w_{0}^{*}\right), & i^{*}=2
\end{array} .\right.
$$

Let us consider problem (56.1) first. Our approach to solving it will be to minimize the system-wide costs and to allow the vendor to yield all the benefits from doing so, leaving the purchaser at the maximum acceptable cost level. Note that (56.1) assumes the no-collection policy; hence, the system-wide optimisation would require adopting the classic joint economic lot size - expressed as $\mathbf{J E L S}_{\mathbf{0}}=q^{*}(0)$ (cf. Sections 3 and 4.3). The vendor can influence the purchaser's choice of order size through the contract parameter $w_{0}$, as equation (48) suggests. Hence, by setting the purchaser's order size $q_{p}^{*}\left(0, w_{0}\right)$ to be equal to the joint economic lot size $q^{*}(0)$, we will be able to determine the fixed fee $w_{0}$ that improves the supply-chain performance in (56.1) to the greatest possible extent. The equation

$$
\sqrt{\frac{2 D \cdot\left(s_{p}+w_{0}\right)}{h_{p}}}=\sqrt{\frac{2 D \cdot\left(s_{p}+s_{v}\right)}{h_{p}+h_{v} \frac{D}{P_{M}}}}
$$

therefore yields after a straightforward transformation the fixed fee

$$
w_{0}^{A}=\frac{s_{v} h_{p} P_{M}-s_{p} h_{v} D}{h_{p} P_{M}+h_{v} D}
$$

(see also Kohli and Park 1989, p. 704). Note that $w_{0}^{A}<0$ holds whenever

$$
\frac{s_{v} h_{p}}{s_{p} h_{v}}<\frac{D}{P_{M}}
$$

which is exactly the condition required for the joint economic lot size to be less than the purchaser's economic order quantity (see also Banerjee 1986a, p. 299). In other words, in that case the fixed fee will effectively reduce the purchaser's fixed ordering cost and encourage him or her to deviate towards a lower order size.

While making an offer to the purchaser, the vendor has to make sure that the purchaser is made neither worse off (to keep contracting feasible) nor better off (to collect all of the benefit and so achieve minimisation in (56.1)). In other words, constraint (55a) has to hold as equality, which yields after a straightforward transformation the following per-unit charge:

$$
w_{1}^{A}=\bar{w}-\frac{\sqrt{s_{p}+w_{0}^{A}}-\sqrt{s_{p}}}{\sqrt{D /\left(2 h_{p}\right)}}=\bar{w}-\frac{1}{D} \cdot\left[\frac{1}{2} \mathbf{J} \mathbf{E L S}_{\mathbf{0}}-\frac{1}{2} \mathbf{E} \mathbf{O Q} \mathbf{Q}_{\mathbf{0}}\right] \cdot h_{p} \cdot 2,
$$

where $\mathbf{J E L S}_{\mathbf{0}}$ is the joint economic lot size as defined above, and $\mathbf{E O Q}_{\mathbf{0}}=q_{p}^{*}(0,0)$ is the purchaser's economic order quantity (cf. Sections 3 and 4.1). Note that the per-unit charge deviates from the original wholesale price by an amount which reflects the shift in the purchaser's inventory-related costs upon acceptance of the fixed fee $w_{0}^{A}$ and the effective transition to the joint economic lot size (cf. discussion following equation (26) in Section 4.1).

To summarise: the deposit amount $d^{A}=0$ and the two-part tariff $w_{0}^{A}, w_{1}^{A}$ represent an optimal solution to problem (56.1) and coordinate the supply chain if the no-collection policy happens to be system-optimal. In particular, the deposit amount guarantees that there will be no collection; the fixed fee is used to incentivise the purchaser to adopt the jointly optimal lot size, and the per-unit charge is used to re-distribute the benefits of that optimisation. 
Turning now to problem (56.2), we can use the same approach as we have just outlined. The difference to the previous problem lies in the fact that, given a deposit amount $d=\theta^{*}\left(w_{0}\right)$, the purchaser will switch to full collection; the purchaser's status quo is the same as above, however. Then, using the same approach, we can show that the following two-part tariff can solve problem (56.2) (we omit specific details for reasons of space):

$$
\begin{aligned}
w_{0}^{B} & =\frac{s_{v} \tilde{h}_{p}-s_{p} \tilde{h}_{v}}{\tilde{h}_{p}+\tilde{h}_{v}}, \\
w_{1}^{B} & =\bar{w}-\frac{1}{D} \cdot\left[\frac{1}{2} \mathbf{J E L S} \mathbf{S}_{\mathbf{1}} \cdot \tilde{h}_{p}-\frac{1}{2} \mathbf{E} \mathbf{O Q} \mathbf{Q}_{\mathbf{0}} \cdot h_{p}\right] \cdot 2-\frac{k}{D}+d^{B}-a,
\end{aligned}
$$

where $\tilde{h}_{p}=h_{p}+u_{p}$ and $\tilde{h}_{v}=\Delta_{M}+2 \Omega_{M}+V=\left(h_{v}-u_{v}\right) \frac{D}{P_{R}}+2 u_{v}$ represent inventory holding costs accruing under the full-collection policy per unit of product per time unit at the purchaser and the vendor respectively, $\mathbf{J E L S}_{1}=q^{*}(1)$ is the joint economic lot size under full collection, and $d^{B}=\theta^{*}\left(w_{0}^{B}\right)$. We can interpret the contract parameters in a similar way as we did in our analysis above of problem (56.1); in particular, the deposit amount $d^{B}$ enforces the full-collection policy, the fixed fee $w_{0}^{B}$ encourages the purchaser to deviate towards the wholly optimal order size, and the per-unit charge $w_{1}^{B}$ re-distributes the benefit. Note that such a contract will achieve coordination whenever the full-collection policy turns out to be system-optimal. Note also that the deposit amount can be increased above the minimum possible value $d^{B}$ in exchange for a decrease by the same amount of the per-unit charge.

Having obtained the optimal solutions to both problems (56.1) and (56.2), we can compare them so as to obtain the solution to the vendor's contract design problem (53) as outlined by equations (57) to (59). From the above considerations, we arrive at the following:

Proposition 7. Optimal contracting on the deposit amount and a two-part tariff yields coordination if and only if a trivial collection rate is optimal for the entire supply chain.

Indeed, the choice between the solutions to problems (56.1) and (56.2) will by construction lead to system-optimal lot-sizing under either the no-collection or the full-collection policy whichever triggers the lower system-wide costs. Hence, supply chain coordination will be achieved provided that a trivial collection rate is optimal from the supply-chain perspective. However, the supply chain will lack coordination whenever the (uniquely defined) systemoptimal collection rate happens to be non-trivial.

\subsection{Numerical analysis}

The previous sections have shown that neither deposit-based control (Section 5.1) nor contracting on the deposit amount and a two-part tariff (Section 5.2) are genrally capable of coordinating the supply chain under consideration. In the present section, we conduct a numerical study to develop insights into the relative performance of both types of control and the capability of the latter to reduce the coordination deficit of the former. We conduct a $3^{k}$ full-factorial study where $k=14$ represents the number of model parameters which are subject to variation. Specifically, each of the model parameters has been set to vary in a range with three possible values (low, medium, high), which has resulted in 4,782,969 distinct combinations of parameter values. Table 1 reports the ranges defined for the individual parameters. Note that we followed Teunter and van der Laan (2002) in setting the holding cost rates for nonserviceables. Note also that the range of parameter $P_{R}$ was set following Proposition A.1 (cf. Section A.4.1 in the Appendix) in such a way that the MbR setting 


\begin{tabular}{|c|c|c|c|c|c|c|c|c|c|c|c|c|c|c|}
\hline \multicolumn{6}{|c|}{ Parameter (vendor) } & \multicolumn{9}{|c|}{ Parameter (purchaser) } \\
\hline Value & $s_{v}$ & $c_{M}$ & $c_{R}$ & $P_{M}$ & $P_{R}^{\dagger, \ddagger}$ & $\alpha_{v}^{\dagger}$ & $o_{v}^{\dagger}$ & $\bar{w}$ & $s_{p}$ & $\alpha_{p}^{\dagger}$ & $o_{p}^{\dagger}$ & $D$ & $a$ & $k$ \\
\hline High & 800 & 30 & $.99 c_{M}$ & $5 D$ & $3 r P_{M}$ & .5 & $.15 c_{M}$ & $1.5 c_{M}$ & 200 & .5 & $0.15 c_{M}$ & 2000 & $.5\left(c_{M}-c_{R}\right)$ & $a D$ \\
\hline Medium & 500 & 20 & $.66 c_{M}$ & $3 D$ & $2 r P_{M}$ & .3 & $.1 c_{M}$ & $1.25 c_{M}$ & 120 & .3 & $0.1 c_{M}$ & 1500 & $.3\left(c_{M}-c_{R}\right)$ & $.75 a D$ \\
\hline Low & 200 & 10 & $.33 c_{M}$ & $D$ & $r P_{M}$ & .1 & $.05 c_{M}$ & $c_{M}$ & 40 & .1 & $0.05 c_{M}$ & 1000 & $.1\left(c_{M}-c_{R}\right)$ & $.5 a D$ \\
\hline
\end{tabular}

Inventory holding costs are comprised of costs of capital and storage (out-of-pocket) costs and determined here accordingly as follows: $h_{v}=\alpha_{v} c_{M}+o_{v}, u_{v}=\alpha_{v} \cdot\left(c_{M}-c_{R}\right)+0.5 o_{v}, h_{p}=\alpha_{p} \bar{w}+o_{p}, u_{p}=\alpha_{p} \cdot\left(c_{M}-c_{R}\right)+0.5 o_{p}$ (cf. Silver et al. 1998, Ch. 3.6.1, and Teunter and van der Laan 2002).

$¥=h_{v} /\left(h_{v}-u_{v}\right)$

Tab. 1: Definition of value ranges for the model parameters in the numerical study

happens to be at least as good as or preferable to the RbM setting. Each combination of parameter values thus specifies a model instance for which we have evaluated the optimal deposit-based contract (DC) of Section 5.1 and the optimal contract on the deposit amount and a two-part tariff (D2PTC) of Section 5.2. The respective system-wide costs under each contract were then compared with the optimal system-wide costs under the centralized supply-chain control as per Section 4.3. This analysis has led us to the following insights. Under DC, the cost penalty compared to the respective supply chain optimum (coordination deficit) averaged $6.3 \%$ over all model instances, with the maximum penalty being as high as $67.8 \%$. Under D2PTC, full coordination is achieved in all those cases (about $83 \%$ of all model instances) when a trivial collection rate is optimal from the supply-chain perspective - in line with the analysis of Section 5.2. Therefore, only in those instances where the systemoptimal collection rate happens to be non-trivial (about 17\% of all model instances) does this contract exhibit an average coordination deficit of $0.12 \%$ and a maximum one of $2 \%$.

We need to point out that the results reported here strongly depend on the choice of value ranges for the individual parameters. We have nevertheless observed in a series of repeated experiments with alternative specifications of value ranges (not reported here) that the comparison between the above results is generally sustained and that contracting on the deposit amount and a two-part tariff thus significantly reduces the coordination deficit of the deposit-based control (both the average and the maximum one) and makes the supply-chain performance on average closely match its performance under the centralized control. Future research should develop deeper insights into this topic.

\subsection{Contracting on the deposit amount and a three-part tariff}

The previous sections have developed insights into the coordination deficit that may persist when contracting occurs either on the deposit amount or its combination with a two-part tariff. In this section, we add some further complexity to the latter contract and show that coordination can always be achieved under this new contract form. As in Savaskan et al. (2004), we need to make certain contract parameters contingent on the collection rate. However, there are differences between the form of the coordinating contract outlined here and that in Savaskan et al. (2004). We summarise these differences at the end of this section.

To arrive at the form of a coordinating contract, consider the purchaser's total cost function under the contract with a deposit $d$ only — as given by equations (1) to (2):

$$
T C_{p}(d, q, \beta)=s_{p} \frac{D}{q}+\left(h_{p}+\beta u_{p}\right) \frac{q}{2}+k \beta+(a-d) \beta D .
$$

Consider also the total cost function of the entire supply chain as defined by (11) to (13): 
$T C_{T}(q, \beta)=\left(s_{v}+s_{p}\right) \frac{D}{q}+\frac{q}{2} \cdot\left\{\Delta_{M} \beta^{2}+\left(2 \Omega_{M}+u_{p}\right) \beta+V+h_{p}\right\}+k \beta+\left(a+c_{R}-c_{M}\right) \beta D+c_{M} D$

(note that the reward parameter $d$ is not relevant from the supply-chain perspective). It is then clear that:

- by making the purchaser internalise the individual components of the vendor's own costs - i.e., setup, holding, manufacturing and costs of returns, and

- compensating the purchaser for the resulting increase in the total costs caused by such cost internalisation,

the vendor would encourage the purchaser to adopt the collection rate and the order size which are optimal from the supply-chain perspective, and thereby achieve supply-chain coordination. Furthermore, the purchaser's total costs would remain unaffected, which would thereby minimise the vendor's total costs to the greatest possible extent.

To this end, consider a scenario in which the vendor offers the purchaser a contract with a three-part tariff $\left(w_{0}, w_{1}, w_{2}\right)$ such that the total payment per order quantity $q$ is calculated as

$$
w(q)=w_{0}+w_{1} q+w_{2} q^{2},
$$

so that the purchaser's payment per time unit amounts, on average, to

$$
\frac{D}{q} \cdot\left(w_{0}+w_{1} q+w_{2} q^{2}\right)=D \cdot\left(\frac{w_{0}}{q}+w_{2} q+w_{1}\right) .
$$

As in Section 5.2, we can now adjust the purchaser's cost function to include the relevant procurement costs (cf. (44)):

$$
T C_{p}\left(d, q, \beta, w_{0}, w_{1}, w_{2}\right)=\left(s_{p}+w_{0}\right) \frac{D}{q}+\left(h_{p}+\beta u_{p}+2 w_{2} D\right) \frac{q}{2}+k \beta+(a-d) \beta D+w_{1} D .
$$

We naturally assume that (70) is defined for all $w_{0}>-s_{p}$, all $w_{1}$, and all $w_{2}$ such that $w_{2}>-\left(h_{p}+\beta u_{p}\right) /(2 D)$. We can then express the purchaser's minimum total costs (15) assuming an optimal choice of the order size as follows (cf. (45)):

$$
T C_{p}^{*}\left(d, \beta, w_{0}, w_{1}, w_{2}\right)=\sqrt{2 D \cdot\left(s_{p}+w_{0}\right)\left(h_{p}+\beta u_{p}+2 w_{2} D\right)}+k \beta+(a-d) \beta D+w_{1} D .
$$

Consider then the following choice by the vendor of the tariff parameters $w_{0}, w_{2}$ :

$$
\begin{aligned}
& w_{0}^{* *}=s_{v}, \\
& w_{2}^{* *}(\beta)=\frac{1}{2 D}\left(\Delta_{M} \beta^{2}+2 \Omega_{M} \beta+V\right) .
\end{aligned}
$$

Note that the latter parameter has been made contingent on the collection rate $\beta$. Consider also the following deposit refund to be offered by the vendor to the purchaser:

$$
d^{* *}=c_{M}-c_{R} .
$$

Then, obviously, as long as the purchaser accepts a contract with the above parameter values, his or her total costs (70) will coincide with $T C_{T}(q, \beta)$, i.e., with those of the entire supply chain, up to the terms $w_{1} D$ and $c_{M} D$ respectively, which are independent of $q$ and $\beta$. Therefore, the purchaser's choice of the optimal order size and collection rate will be guided by equation (18) and Proposition 3 respectively, thus leading to supply-chain coordination. The vendor should, however, choose the tariff parameter $w_{1}$ (the per-unit charge) so that, in accepting the contract, the purchaser is made neither worse off (to keep contracting feasible) 
nor better off (to collect all of the benefit) - compared to the case of deposit-only control analysed in Section 5.1. Therefore, the per-unit charge $w_{1}$ has to satisfy (cf. (43), (46)):

$$
T C_{p}^{*}\left(d^{* *}, \beta_{T}^{*}, w_{0}^{* *}, w_{1}, w_{2}^{* *}\left(\beta_{T}^{*}\right)\right)=T C_{p}^{*}\left(d_{v}^{*}, \beta_{p}^{*}\left(d_{v}^{*}, 0\right), 0, \bar{w}, 0\right),
$$

where $\bar{w}$ is, as before, the wholesale price prior to the introduction of the tariff. Note that, in (43), the vendor's deposit-only choice $d_{v}^{*}$ can be either 0 or $\theta^{*}(0)$; we can, though, immediately verify that either choice implies the same total costs for the purchaser (cf. the discussion in Section 5.2 following problem (53)):

$$
T C_{p}^{*}\left(0, \beta_{p}^{*}(0,0), 0, \bar{w}, 0\right)=T C_{p}^{*}\left(\theta^{*}(0), \beta_{p}^{*}\left(\theta^{*}(0), 0\right), 0, \bar{w}, 0\right)=\sqrt{2 D s_{p} h_{p}}+\bar{w} D .
$$

We can then express the required per-unit charge from (75) as follows:

$$
w_{1}^{* *}=\bar{w}-\frac{1}{D} \cdot\left[\frac{1}{2} q^{*}\left(\beta_{T}^{*}\right) H\left(\beta_{T}^{*}\right)-\frac{1}{2} \mathbf{E} \mathbf{O Q} \mathbf{Q}_{0} \cdot h_{p}\right] \cdot 2-\frac{k}{D} \beta_{T}^{*}+\left(d^{* *}-a\right) \beta_{T}^{*},
$$

where $q^{*}\left(\beta_{T}^{*}\right)$ is the joint economic lot size under the system-optimal collection rate $\beta_{T}^{*}$, $\mathbf{E} \mathbf{O Q} \mathbf{Q}_{\mathbf{0}}=q_{p}^{*}(0)$ is the purchaser's economic order quantity, and $H(\beta)=\Delta_{M} \beta^{2}+U \beta+W$ is the system-wide holding cost per unit of product per time unit, assuming collection rate $\beta$ (cf. Sections 2.4 and 3). Thus, similar to the analysis that we conducted in Section 5.2, the per-unit charge $w_{1}^{* *}$ is intended to re-distribute the efficiency gain achieved by adopting contract parameters (72) to (74). Note that it deviates from the original wholesale price by an amount which reflects, firstly, the shift in the purchaser's inventory-related costs upon the transition to the system-optimal collection rate and joint economic lot size (cf. discussion following eq. (26) in Section 4.1), and, secondly, the associated costs of returns acquisition.

It is interesting to obtain the following insight into the structure of the three-part tariff (68): equation (69) shows that the price paid per unit can be separated into the invariant perunit charge $w_{1}$ and the fee that depends on the order size $q$. In contrast to the two-part tariff of Section 5.2, where the procurement price fell (grew) in $q$ with a positive (negative) value of $w_{0}$, we can see in the current case that the effect exercised in the same way by the parameter $w_{0}$ is now moderated by the parameter $w_{2}$, so that (assuming $w_{0}, w_{2}>0$ - as is the case in (72) to (73)), infinitely increasing $q$ does not lead to a monotone decrease in the price; instead, there is a finite quantity $\breve{q}=2 \sqrt{w_{0} w_{1}}$, which would minimise the procurement price, should it be considered separately from all other costs involved.

Comparing now the coordinating contract (72) to (74), (77) with the one obtained by Savaskan et al. (2004) in their setting, we can see that a two-part tariff was sufficient in their case to achieve coordination, whereas we needed to resort to a three-part tariff. Furthermore, their optimal contract makes the deposit unnecessary, whereas the deposit amount (74) needs to be retained in our setting. ${ }^{3}$ However, what is common to both our and their optimal contract is that one of the contract parameters is made contingent on the collection rate.

\section{Discussion and conclusions}

We have studied a complex of problems addressing the individual, the system-optimal and the decentralised control in a closed-loop supply chain with a single vendor and a single purchaser. Our modelling approach captures operational aspects of such a supply chain while also addressing the coordination problem. We were able to obtain closed-form solutions to the problems under study, making possible their interpretation and facilitating their evaluation. 
We have observed that the individual preferences of supply-chain members with regard to collecting and remanufacturing used products can be quite different: while the purchaser always prefers a pure collection policy (i.e., either no collection or full collection), there are conditions under which the vendor always prefers a mixed policy. Also, when both parties prefer a pure policy, their preferences with regard to it may still diverge. Furthermore, the optimal collection policy from the supply-chain perspective may or may not be a pure one. Considering the decentralised control in this supply chain, we have seen that supply-chain coordination is unlikely to occur if the vendor employs the deposit as the only means to influence the purchaser's decisions. This, however, changes when the parties agree to determine contract parameters in a cooperative fashion and settle on a deposit amount with a two-part tariff via bargaining. This leads to supply-chain coordination whenever a pure collection policy is optimal for the entire supply chain. Nevertheless, coordination cannot be achieved if a mixed collection policy happens to be optimal from the supply-chain perspective. We nonetheless pursued a further analysis of this contract form to determine its optimal implementation in the closed-loop context should the user prefer to stick to it given the capability of the two-part tariff to coordinate the "traditional" (forward) supply chain. The closed-form solution thus obtained provided qualitative insights into the coordination capability of this contract form and facilitated evaluation of the persisting coordination deficit in a numerical study - which has revealed a substantial reduction of the coordination deficit compared to the deposit-based control. Developing further insights into this capability of this contract form represents an interesting question for future research. We have also been able to establish the form of coordinating contract that features a novel threepart tariff and a deposit; however, it is up to the user to decide whether this contract form or contracting explicitly on the system-optimal lot size and collection rate - as in Banerjee (1986a) - is more preferable in practice.

It must be noted that several simplifying assumptions have been made in our study which provided for the analytical tractability of the problems addressed. Firstly, we rely on the assumptions of the conventional joint economic lot size model, which treats demand and lead time as deterministic constant (cf. Banerjee 1986a). Secondly, the lot-for-lot assumption underlying that model precludes stock-holding at the vendor between two consecutive shipments to the purchaser - which does not necessarily represent an optimal policy (cf. Ben-Daya et al. 2008). Thirdly, we assume a joint setup cost for manufacturing and remanufacturing operations within a cycle at the vendor - something that does not, however, represent a general case (cf. Teunter et al. 2006). Fourthly, following the lot-for-lot assumption, we restrict the production policy at the vendor to having at most one manufacturing and one remanufacturing batch within a cycle at the vendor; however, the question regarding the form of the optimal policy in more general settings remains (cf. Konstantaras and Skouri 2010). Fifthly, we resort to a simplified form of the collection effort function adopted in Savaskan et al. (2004) — which represents a crude approximation. Hence, the optimality of solutions obtained must be understood within the context of the assumptions made; these solutions may lose their optimality if these assumptions are relaxed or do not hold. Relaxing these assumptions indicates several interesting opportunities for future research, and we could also extend our study to account for price-sensitive demand (as in Savaskan et al. (2004) and Liu et al. (2009)) and consumer behaviour (as in Zeng (2013)).

\section{Notes}

${ }^{1}$ This dependence can be directly generalized to the form $\beta=I / k+b-$ which, on the one hand, offers a better approximation of the square-root function $\beta=\sqrt{I / k}$ assumed in 
Savaskan et al. (2004), but, on the other, implies that a certain fraction of used items, represented by the intercept $b$, return without the collector's effort. This may still represent a realistic situation in the presence of educated customers who, for example, leave end-of-use products at dedicated locations. To simplify the subsequent presentation, we assume $b=0$; in this case, $\beta=0$ if $I=0$ as well as $\beta=1$ if $I=k$, representing respectively the cases of $n o$ collection and full collection. The subsequent analysis can obviously be generalised to the case of $b>0$, leading to the minimum value of the collection rate $\beta_{\min }=b$, and its maximum value can be restricted to $\beta_{\max }<1$; in these cases, we should speak of no and full collection effort respectively.

${ }^{2}$ It would be sufficient to require $\beta P_{R}+(1-\beta) P_{M} \geq D$ to ensure that the vendor is capable of meeting the entire demand of the purchaser. To ensure the same for all possible remanufacturing rates $\beta \in[0,1], P_{R}, P_{M} \geq D$ is required, though. Most of our results will hold under this assumption, too, or can otherwise be adjusted in a straightforward way to accommodate it. To streamline the subsequent analysis and its interpretation, we will maintain the assumption $P_{R}, P_{M}>D$.

${ }^{3}$ Alternatively, we could make the per-unit charge $w_{1}$ contingent on the collection rate in order to eliminate the deposit payments from the contract.

\section{Acknowledgements}

The authors would like to thank two anonymous referees and Professor Peter Recht of TU Dortmund University for their many constructive comments on an earlier version of this paper. Imre Dobos gratefully acknowledges the financial support of the OTKA-105888 research programme and the Deutscher Akademischer Austauschdienst (DAAD). Knut Richter acknowledges the support of the Sankt Petersburg University in enabling him to continue his work on the area of closed-loop supply-chain management.

\section{References}

Affisco JF, Paknejad MJ, Nasri F (2002) Quality improvement and setup reduction in the joint economic lot size model. Eur J Oper Res 142:497-508

Akçalı E, Çetinkaya S (2011) Quantitative models for inventory and production planning in closed-loop supply chains. Int J Prod Res 49:2373-2407

Atasu A, Çetinkaya S (2006) Lot sizing for optimal collection and use of remanufacturable returns over a finite life-cycle. Prod Oper Manag 15:473-487

Atasu A, Guide VDR, Van Wassenhove LN (2008) Product reuse economics in closed-loop supply chain research. Prod Oper Manag 17:483-496

Atasu A, Guide VDR, Van Wassenhove LN (2010) So what if remanufacturing cannibalizes my new product sales? Calif Manage Rev 52:56-76

Atasu A, Toktay LB, Van Wassenhove LN (2013) How collection cost structure drives a manufacturer's reverse channel choice. Prod Oper Manag 22: 1089-1102

Banerjee A (1986a) A joint economic lot-size model for purchaser and vendor. Decision Sci 17:292-311

Banerjee A (1986b) On “A quantity discount model to increase vendor profits”. Manage Sci 32:1513-1517

Ben-Daya M, Darwish M, Ertogral K (2008) The joint economic lot sizing problem: Review and extensions. Eur J Oper Res 185:726-742 
Cachon GP (2003) Supply chain coordination with contracts. In: de Kok AG, Graves SC (eds) Supply Chain Management: Design, Coordination and Operation. Elsevier, pp 229-339

Cai C (2011) Quantity discounts contract coordination model of three-stage closed-loop supply chain under retailer price competition. In: Proceedings of 2011 International Conference on Transportation, Mechanical, and Electrical Engineering, December 16-18, 2011, Changchun, China, IEEE, pp 195-199

Corbett CJ, Savaskan RC (2002) Contracting and coordination in closed-loop supply chains. In: Guide VDR, Van Wassenhove LN (eds) Closed-loop Supply Chains: A Business Perspective, Carnegie Bosch Institute, Pittsburgh, PA, pp 93-113

Dobos I, Richter K (2004) An extended production/recycling model with stationary demand and return rates. Int J Prod Econ 90:311-323

Dobos I, Gobsch B, Pakhomova N, Pishchulov G, Richter K (2011a) A vendor-purchaser economic lot size problem with remanufacturing and deposit. Discussion Paper 304, Faculty of Economics and Business Administration, European University Viadrina, Frankfurt (Oder), Germany

Dobos I, Gobsch B, Pakhomova N, Richter K (2011b) Remanufacturing of used products in a closed-loop supply chain. In: Csutora M, Kerekes S (eds) Accounting for climate change - What and how to measure. Proceedings of the EMAN-EU 2011 Conference, 24-25 January 2011, Budapest, Hungary, pp $130-146$

Dobos I, Gobsch B, Pakhomova N, Pishchulov G, Richter K (2013) Design of contract parameters in a closed-loop supply chain. Cent Eur J Oper Res 21:713-727

Dolan RJ (1987) Quantity discounts: Managerial issues and research opportunities. Marketing Sci 6:1-22

Govindan K, Popiuc MN (2013) Reverse supply chain coordination by revenue sharing contract: A case for the personal computers industry. Eur J Oper Res 233:326-336

Guide VDR, Van Wassenhove LN (2009) The evolution of closed-loop supply chain research. Oper Res $57: 10-18$

Hong X, Wang Z, Wang D, Zhang H (2013) Decision models of closed-loop supply chain with remanufacturing under hybrid dual-channel collection. Int J Adv Manuf Technol 68:1851-1865

Jaber MY, El Saadany AMA (2011) An economic production and remanufacturing model with learning effects. Int J Prod Econ 131:115-127

Jonrinaldi, Zhang DZ (2013) An integrated production and inventory model for a whole manufacturing supply chain involving reverse logistics with finite horizon period. Omega 41:598-620

Jun Y, Susheng W (2011) Optimal contract design of reverse supply chain considering uncertain recycle price. In: Proceedings of 2011 International Conference on E -Business and E -Government, May 6-8, 2011, Shanghai, China, IEEE, pp 1419-1423

Kohli R, Park H (1989) A cooperative game theory model of quantity discounts. Manage Sci 35:693-707

Konstantaras I, Skouri K (2010) Lot sizing for a single product recovery system with variable setup numbers. Eur J Oper Res 203:326-335

Liu X, Banerjee A, Kim S-L (2009) Models for retail pricing and customer return incentive for remanufacturing a product. In: Proceedings of the POMS 20th Annual Conference, Orlando, Florida, USA, May 1 to 4, 2009

Liu X, Çetinkaya S (2007) A note on "quality improvement and setup reduction in the joint economic lot size model". Eur J Oper Res 182:194-204

Ma W, Sun J (2012) Research for closed-loop supply chain price models under uncertain demand. In: Proceedings of 2012 Second International Conference on Business Computing and Global Informatization, 12-14 October 2012, Shanghai, China, IEEE, pp 136-139

Matsumoto M, Umeda Y (2011) An analysis of remanufacturing practices in Japan. J Remanuf 1:2

Minner S, Lindner G (2004) Lot sizing decisions in product recovery management. In: Dekker R, Fleischmann M, Inderfurth K, Van Wassenhove LN (eds) Reverse logistics: Quantitative models for closed-loop supply chains, Springer, New York, pp 157-180

Monahan JP (1984) A quantity discount pricing model to increase vendor profits. Manage Sci 30:720-726

Myerson R (1983) Mechanism design by an informed principal. Econometrica 51:1767-1797

Nie J, Huang Z, Zhao Y, Shi Y (2013) Collective recycling responsibility in closed-loop fashion supply chains with a third party: Financial sharing or physical sharing? Math Probl Eng 2013:1-11 
Pibernik R, Zhang Y, Kerschbaum F, Schröpfer A (2011) Secure collaborative supply chain planning and inverse optimization - The JELS model. Eur J Oper Res 208:75-85

Pishchulov G, Dobos I, Gobsch B, Pakhomova N, Richter K (2012) Remanufacturing of used products in a closed-loop supply chain with quantity discount. In: Klatte D, Lüthi HJ, Schmedders K (eds) Operations Research Proceedings 2011: Selected Papers of the International Conference on Operations Research (OR 2011), August 30-September 2, 2011, Zurich, Switzerland, Springer, pp 457-462

Reyniers DJ (2001) The effect of vertical integration on consumer price in the presence of inventory costs. Eur J Oper Res 130:83-89

Richter K (1994) An EOQ repair and waste disposal model. In: Proceedings of the Eighth International Working Seminar on Production Economics, Austria, February 1994, vol. 3, pp 83-91

Richter K (1996a) The variable EOQ repair and waste disposal model with variable setup numbers. Eur J Oper Res 96:313-324

Richter K (1996b) The extended EOQ repair and waste disposal model. Int J Prod Econ 45:443-447

Ross SA (1973) The economic theory of agency: The principal's problem. Am Econ Rev 63:134-139

Rubio S, Chamorro A, Miranda FJ (2008) Characteristics of the research on reverse logistics (1995-2005). Int J Prod Res 46:1099-1120

Savaskan RC, Bhattacharya S, Van Wassenhove LN (2004) Closed-loop supply chain models with product remanufacturing. Manage Sci 50:239-252

Savaskan RC, Van Wassenhove LN (2006) Reverse channel design: The case of competing retailers. Manage Sci 52:1-14

Schrady DA (1967) A deterministic inventory model for reparable items. Nav Res Logist Q 14:391-398

Shi C, Bian D, Zhang H (2010) Loss-averse closed-loop supply chain coordination by revenue sharing contract and quantity discount contract. In: Proceedings of $20102^{\text {nd }}$ International Conference on Computer Engineering and Technology, Zibo, China, 16-18 April 2010, IEEE, vol. 1, pp 365-369

Silver EA, Pyke DF, Peterson R (1998) Inventory Management and Production Planning and Scheduling, $3^{\text {rd }}$ edn. Wiley, New York

Steinhilper R (1998) Remanufacturing: The Ultimate Form of Recycling. Fraunhofer IRB Verlag, Stuttgart

Sucky E (2006) A bargaining model with asymmetric information for a single supplier-single buyer problem. Eur J Oper Res 171:516-535

Tang O, Teunter RH (2006) Economic lot scheduling problem with returns. Prod Oper Manag 15: 488-497

Teunter RH (2001) Economic ordering quantities for recoverable item inventory systems. Nav Res Log 48:484-495

Teunter R (2004) Lot-sizing for inventory systems with product recovery. Comput Ind Eng 46:431-441

Teunter RH, Bayindir ZP, Van Den Heuvel W (2006) Dynamic lot sizing with product returns and remanufacturing. Int J Prod Res 44:4377-4400

Teunter R, van der Laan E (2002) On the non-optimality of the average cost approach for inventory models with remanufacturing. Int J Prod Econ 79:67-73

Wei J, Zhao J, Li Y (2012) Pricing decisions for a closed-loop supply chain in a fuzzy environment. Asia Pac J Oper Res 29(1):1240003-1-30

Xu X, Li Y, Cai X (2012) Optimal policies in hybrid manufacturing/remanufacturing systems with random price-sensitive product returns. Int J Prod Res 50:6978-6998

Yan N, Sun B (2012) Optimal Stackelberg strategies for closed-loop supply chain with third-party reverse logistics. Asia Pac J Oper Res 29(5):1250026-1-11

Zeng AZ (2013) Coordination mechanisms for a three-stage reverse supply chain to increase profitable returns. Nav Res Log 60:31-45

Zhao X, Zhao X (2011) Pricing decision and performance analysis of closed-loop supply chain with thirdparty reverse logistics. In: Proceedings of 2011 International Conference on Management and Service Science, Wuhan, China, 12-14 August 2011, IEEE, pp 1-4 


\section{Appendix}

\section{A.1 Proof of Lemma 1}

Proof: As discussed in Section 4, the assumption $A B>C^{2}$ of the Lemma implies that $K(\beta)$ is strictly convex. By the strict convexity, $K^{\prime \prime}(\beta)>0$ holds, and thus $K^{\prime}(\beta)$ is monotone increasing at each point of the domain $0 \leq \beta \leq 1$. Conditions $(22 \mathrm{a}, \mathrm{b})$ in turn imply that $K^{\prime}(0)<0$ and $K^{\prime}(1)>0$ hold respectively. We can therefore see that $(22 \mathrm{a}, \mathrm{b})$ cannot be violated simultaneously since this would require both $K^{\prime}(0) \geq 0$ and $K^{\prime}(1) \leq 0$ to hold, which is impossible given the monotonic growth of $K^{\prime}(\beta)$. Hence, at least one of the conditions (22a, b) holds.

Assume now that $(22 \mathrm{a})$ does not hold - which implies $K^{\prime}(0) \geq 0$. Due to its monotonic growth, $K^{\prime}(\beta)>0$ at all $0<\beta \leq 1$, which proves that $K(\beta)$ grows everywhere to the right of $\beta=0$. Then, obviously, $\beta^{*}=0$ is its global minimum. This proves assertion a) of the Lemma. By a similar argument, we can prove assertion $b)$.

Let both $(22 \mathrm{a}, \mathrm{b})$ now hold. Then $K(\beta)$ decreases at $\beta=0$ due to $K^{\prime}(0)<0$ and increases at $\beta=1$ due to $K^{\prime}(1)>0$. By the strict convexity, the global minimum is attained at the unique interior point $\beta^{*} \in(0,1)$ with $K^{\prime}\left(\beta^{*}\right)=0$, which we accordingly determine by solving

$$
K^{\prime}(\beta)=G \frac{B \beta+C}{\sqrt{A+B \beta^{2}+2 C \beta}}+E=0
$$

which implies:

$$
\frac{(B \beta+C)^{2}}{B \beta^{2}+2 C \beta+A}=\frac{E^{2}}{G^{2}} \text {. }
$$

Note further that $B>0$ holds due to the assumption of the Lemma. Then (A2) can be rewritten in the following form:

$$
\frac{(B \beta+C)^{2}}{(B \beta+C)^{2}+A B-C^{2}}=\frac{E^{2}}{B G^{2}} .
$$

Denoting

$$
\gamma=B \beta+C
$$

gives:

$$
\frac{\gamma^{2}}{\gamma^{2}+A B-C^{2}}=\frac{E^{2}}{B G^{2}} .
$$

Note that $A B-C^{2}>0$ holds by assumption of the Lemma. Hence, by virtue of (A5), $B G^{2}>E^{2}$ necessarily holds, yielding the inequality $B G^{2}-E^{2}>0$. Then (A5) can be rewritten as follows after rearranging the terms:

$$
\gamma^{2}=E^{2} \cdot \frac{A B-C^{2}}{B G^{2}-E^{2}}
$$

The roots of this equation are given by

$$
\gamma_{1,2}= \pm|E| \cdot \sqrt{\frac{A B-C^{2}}{B G^{2}-E^{2}}},
$$


so that the roots of equation (A2) are

$$
\beta_{1,2}=-\frac{C}{B} \pm \frac{|E|}{B} \sqrt{\frac{A B-C^{2}}{B G^{2}-E^{2}}} .
$$

Note that (A8) is well-defined since the expression under the radical is guaranteed to be positive due to the assumption of the Lemma and the inequality $B G^{2}-E^{2}>0$. Substituting both roots for $\beta$ in (A1), we can see that the solution to the latter is $\beta^{*}=\beta_{2}$ if $E>0$, otherwise $\beta^{*}=\beta_{1}$. Thus the following applies:

$$
\beta^{*}=-\frac{C}{B}-\frac{E}{B} \sqrt{\frac{A B-C^{2}}{B G^{2}-E^{2}}} .
$$

Note also that inequalities $(22 \mathrm{a}, \mathrm{b})$ cannot simultaneously hold if $A B \leq C^{2}$. Indeed, the latter condition would imply $K^{\prime \prime}(\beta) \leq 0$ and therefore a decline or a constancy of $K^{\prime}(\beta)$ on the entire domain, which makes the inequalities $K^{\prime}(0)<0$ and $K^{\prime}(1)>0$ implied by $(22 \mathrm{a}, \mathrm{b})$ incompatible with each other. This completes the proof of assertion c) of the Lemma.

\section{A.2 Proof of Lemma 2}

Proof: Condition $A B \leq C^{2}$ implies that $K^{\prime \prime}(\beta)$ is either negative on the entire domain $0 \leq \beta \leq 1$, or zero. Hence $K(\beta)$ is either strictly concave or affine. Therefore, if a local optimum is found in the domain's interior, it can only be a global maximum; otherwise, the function is monotonic on the entire domain and, in either case, it is sufficient to compare its values at the boundary points to figure out a global minimum. This leads directly to the assertion of the lemma.

\section{A.3 Proof of Corollary 1}

Proof: Indeed, let $c_{M}-c_{R}-d=0$. By definition of parameters $\Omega_{M}$ and $\Delta_{M}$ in (5), there always holds the inequality $\Omega_{M}+\Delta_{M}>0$. If $\Omega_{M}<0$, then conditions (28a, b) are obviously fulfilled and the vendor's optimal remanufacturing rate is non-trivial according to Proposition 1. Furthermore, it is clear that, by definition of parameter $\Omega_{M}$, the inequality $\Omega_{M}<0$ implies that $P_{R}>P_{M}$ holds.

Let now $\Omega_{M} \geq 0$. Then condition (28a) does not hold and thus, according to Proposition 1 , the vendor's optimal remanufacturing rate is 0 or 1 . We will show that the latter cannot occur. Indeed, by comparing the vendor's minimum total costs (17) at $\beta=0$ and $\beta=1$, we can see that $\beta^{*}=1$ if and only if

$$
\sqrt{V+\Delta_{M}+2 \Omega_{M}}-\sqrt{V} \leq 0 .
$$

However, this inequality cannot be fulfilled given that both $\Omega_{M} \geq 0$ and $\Omega_{M}+\Delta_{M}>0$ hold. This completes the proof. 


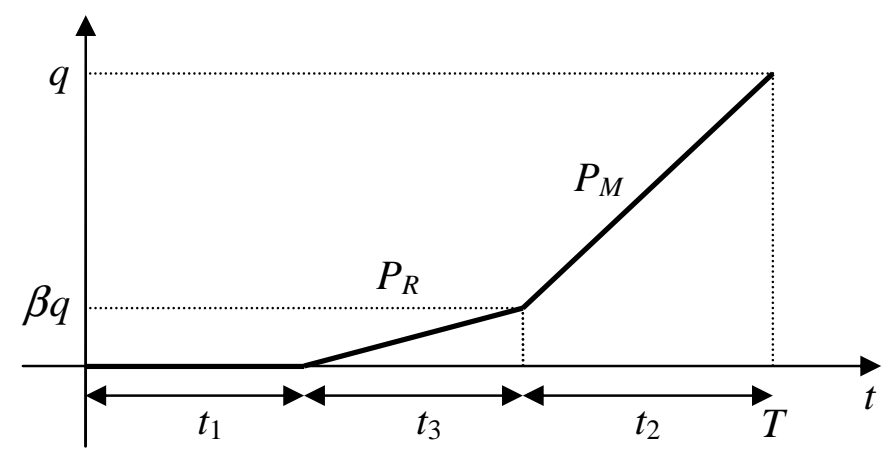

Fig. A.1: The inventory level of serviceables at the vendor during an order cycle

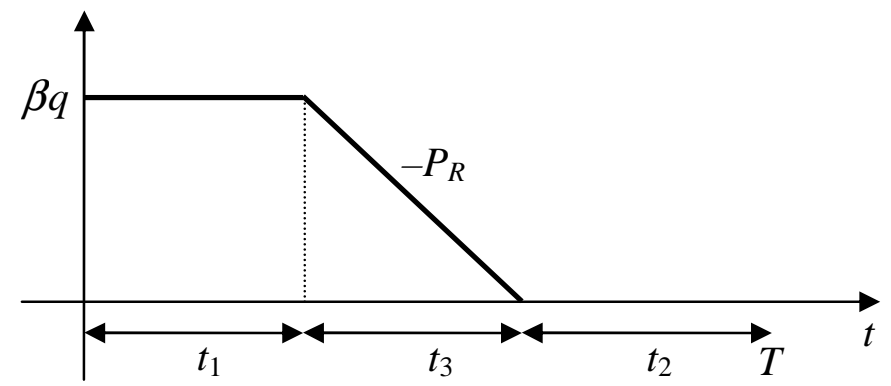

Fig. A.2: The inventory level of nonserviceables at the vendor during an order cycle

\section{A.4 Analysis for the remanufacturing before manufacturing (RbM) setting}

\section{A.4.1 Vendor's inventory costs}

Due to the assumption that remanufacturing precedes manufacturing, the inventory levels of serviceables and nonserviceables at the vendor evolve during an order cycle as illustrated in Figures A.1 and A.2 respectively. The vendor's total inventory holding costs per time unit can be expressed as follows:

$$
\begin{aligned}
& H_{s}(q, \beta)+H_{n}(q, \beta)=\frac{q}{2} \cdot\left\{h_{v} \cdot\left[\beta^{2} \cdot\left(\frac{D}{P_{R}}-\frac{D}{P_{M}}\right)+\frac{D}{P_{M}}\right]+u_{v} \cdot\left[\beta^{2} \cdot\left(2 \cdot \frac{D}{P_{M}}-\frac{D}{P_{R}}\right)+\beta \cdot\left(2-2 \cdot \frac{D}{P_{M}}\right)\right]\right\}= \\
& =\frac{q}{2} \cdot\left\{h_{v} \cdot\left[\beta^{2} \frac{D}{P_{R}}+\left(1-\beta^{2}\right) \frac{D}{P_{M}}\right]+u_{v} \beta \cdot\left[2-2(1-\beta) \frac{D}{P_{M}}-\beta \frac{D}{P_{R}}\right]\right\} .
\end{aligned}
$$

By comparing the vendor's holding cost expressions (5) to (6) and (A11) in MbR and RbM settings respectively, we can obtain the following:

Proposition A.1. For any order size $q$ and remanufacturing rate $\beta$, the following holds:

1. The vendor's total holding costs in the MbR setting are strictly lower than those in the RbM setting if and only if

$$
\frac{P_{R}}{P_{M}}>1+\frac{u_{v}}{h_{v}-u_{v}} .
$$

2. They are equal if and only if (A12) holds as equality or $\beta \in\{0,1\}$.

Hence the MbR setting outperforms the $\mathrm{RbM}$ setting if the production rate of remanufacturing sufficiently exceeds that of manufacturing; otherwise the RbM setting proves to be more 
economical. Note however that the assumptions underlying our model exclude possible raw material stock from our consideration - or, equivalently, consider the raw materials to be shipped to supply the manufacturing process just-in-time. This may not always be realistic due to economies of scale in procurement, there may therefore exist a stock of raw materials at the beginning of the cycle - which would make the RbM option less attractive due to the additional carrying costs. While the assumptions made helped us to simplify the presentation, extending the model to include the raw material stock represents an interesting future research opportunity. Note also that (A12) represents a stronger condition than (31), which is necessary for the vendor's optimal remanufacturing rate in the MbR setting to be non-trivial.

\section{A.4.2 Vendor's solution (problem VC-RbM)}

As in Section 2.3, define

$$
\Delta_{R}=\left(h_{v}-u_{v}\right)\left(\frac{D}{P_{R}}-\frac{D}{P_{M}}\right)+u_{v} \frac{D}{P_{M}} \quad \text { and } \quad \Omega_{R}=u_{v} \cdot\left(1-\frac{D}{P_{M}}\right) .
$$

Then the vendor's holding costs (A11) can be rewritten as

$$
H_{v}(q, \beta)=\frac{q}{2} \cdot\left\{\Delta_{R} \beta^{2}+2 \Omega_{R} \beta+V\right\},
$$

his or her total costs as

$$
T C_{v}(q, \beta)=s_{v} \frac{D}{q}+\frac{q}{2} \cdot\left\{\Delta_{R} \beta^{2}+2 \Omega_{R} \beta+V\right\}+R_{v}(\beta),
$$

and minimum total costs for a given remanufacturing rate as

$$
T C_{v}^{*}(\beta)=\sqrt{2 D s_{v} \cdot\left(\Delta_{R} \beta^{2}+2 \Omega_{R} \beta+V\right)}+R_{v}(\beta) .
$$

The latter expression represents the function $K(\beta)$ as defined in (20), with $A=V, B=\Delta_{R}$, $C=\Omega_{R}, E=\left(d+c_{R}-c_{M}\right) D, F=c_{M} D$ and $G=\sqrt{2 D s_{v}}$ which obviously complies with assumptions (21). By virtue of Lemmas 1 and 2, and similarly to Section 4.2, there holds:

Proposition A.2. The vendor's optimal remanufacturing rate in the RbM setting is non-trivial and given by

$$
\beta_{v, R b M}^{*}=-\frac{\Omega_{R}}{\Delta_{R}}-\frac{E}{\Delta_{R}} \sqrt{\frac{V \Delta_{R}-\Omega_{R}^{2}}{2 D s_{v} \Delta_{R}-E^{2}}}
$$

whenever both of the following conditions hold:

$$
\frac{\Omega_{R}}{\sqrt{V}}<\frac{\left(c_{M}-c_{R}-d\right) D}{\sqrt{2 D s_{v}}}<\frac{\Omega_{R}+\Delta_{R}}{\sqrt{V+\Delta_{R}+2 \Omega_{R}}},
$$

and is otherwise $\beta_{v, R b M}^{*}=0$ or $\beta_{v, R b M}^{*}=1$.

Proposition A.2 can be interpreted in a similar way as Proposition 2 in Section 4.2, and we omit discussion of it here for reasons of space. It is, however, worth observing that $\Omega_{R}>0$ holds by definition of $\Omega_{R}$ in (A13); hence, in contrast to Corollary 1 of Section 4.2 regarding the MbR setting, there holds the following: 
Corollary A.1. If the vendor's nominal benefit $c_{M}-c_{R}-d$ per unit of nonserviceables is zero or negative, then his or her optimal remanufacturing rate in the RbM setting is never nontrivial.

Corollary A.1 can be proven by taking the same approach that we used to prove Corollary 1; the key argument is that with a non-positive nominal benefit and $\Omega_{R}>0$, condition (A18a) is violated. Note also that, in contrast to the MbR setting, where the zero nominal benefit excludes the optimality of full remanufacturing for the vendor (cf. Corollary 1), it does not do so in the RbM setting but excludes the optimality of a mixed policy for the vendor. As in Section 4.2, we derive here the following corollary for the general case:

Corollary A.2. For the vendor's optimal remanufacturing rate in the RbM setting to be nontrivial, the following conditions must necessarily hold:

$$
\frac{P_{M}}{P_{R}}>1-\frac{u_{v}}{h_{v}-u_{v}} \quad \text { and } \quad d<c_{M}-c_{R} .
$$

Thus, with a relatively low holding cost $u_{v}$ of nonserviceables at the vendor, the production rate of manufacturing must be above a sufficiently large fraction of the production rate of remanufacturing for $\beta_{v, R b M}^{*}$ to be a non-trivial remanufacturing rate. In addition, the vendor's nominal benefit per unit of nonserviceables must be positive.

An interpretation of the vendor's willingness to engage in remanufacturing in the RbM setting can be provided in the same way that we did in Section 4.2 following Corollary 2, and we therefore omit it here for reasons of space.

\section{A.4.3 The total system solution (SC-RbM)}

Assuming the optimal choice of the order size, we can express the system-wide costs in the RbM setting as

$$
T C_{T}^{*}(\beta)=\sqrt{2 D \cdot\left(s_{v}+s_{p}\right)\left(\Delta_{R} \beta^{2}+\left(2 \Omega_{R}+u_{p}\right) \beta+W\right)}+R_{T}(\beta)
$$

and represent $K(\beta)$ as defined in (20) with $A=W=V+h_{p}, B=\Delta_{R}, C=\Omega_{R}+u_{p} / 2$, $E=k+\left(a+c_{R}-c_{M}\right) D, F=c_{M} D$ and $G=\sqrt{2 D \cdot\left(s_{v}+s_{p}\right)}$, which obviously complies with assumptions (21). Then, by virtue of Lemmas 1 and 2, and similarly to Section 4.3, there holds:

Proposition A.3. The system-optimal collection rate in the RbM setting is non-trivial and given by

$$
\beta_{T, R b M}^{*}=-\frac{\Omega_{R}+u_{p} / 2}{\Delta_{R}}-\frac{E}{\Delta_{R}} \sqrt{\frac{\left(V+h_{p}\right) \Delta_{R}-\left(\Omega_{R}+u_{p} / 2\right)^{2}}{2 D \cdot\left(s_{v}+s_{p}\right) \Delta_{R}-E^{2}}}
$$

whenever both of the following conditions hold:

$$
\frac{\Omega_{R}+u_{p} / 2}{\sqrt{V+h_{p}}}<\frac{\left(c_{M}-c_{R}-a\right) D-k}{\sqrt{2 D \cdot\left(s_{v}+s_{p}\right)}}<\frac{\Omega_{R}+\Delta_{R}+u_{p} / 2}{\sqrt{V+\Delta_{R}+2 \Omega_{R}+h_{p}+u_{p}}}
$$

and is otherwise $\beta_{T, R b M}^{*}=0$ or $\beta_{T, R b M}^{*}=1$.

Proposition A. 3 can be interpreted in a similar way as Proposition A.2 in the previous section. Similarly, we obtain here the following: 
Corollary A.3. If the supply chain's nominal benefit $c_{M}-c_{R}-a$ per unit of nonserviceables is equal to or below the promotional effort $k / D$ needed to stimulate the return of that unit from the market, then the optimal collection rate from the supply-chain perspective is never non-trivial.

The obvious proof for Corollary A.3 is the observation that $\Omega_{R}+u_{p} / 2>0$ holds by definition of both parameters involved and leads to violation of condition (A22a) whenever $c_{M}-c_{R}-a \leq k / D$. Furthermore, as with Corollary A.2 in the previous section, in the general case there holds the following:

Corollary A.4. For the supply chain's optimal collection rate in the RbM setting to be nontrivial, the following conditions must necessarily hold:

$$
\frac{P_{M}}{P_{R}}>1-\frac{u_{v}}{h_{v}-u_{v}} \quad \text { and } \quad c_{M}-c_{R}-a>k / D .
$$

Note that conditions (A19a) and (A23a) coincide, and hold whenever $u_{v} \geq h_{v} / 2$, while otherwise being implied by the condition

$$
\frac{P_{R}}{P_{M}}<1+\frac{u_{v}}{h_{v}-u_{v}}
$$

- which, according to Proposition A.1, is necessary and sufficient for RbM to outperform MbR given any order size $q$ and remanufacturing rate $\beta \in(0,1)$, from the perspectives of both the vendor and the supply chain.

Again, the attractiveness of collecting and remanufacturing used products in the RbM setting from the supply-chain perspective can be interpreted in the same way as for the MbR setting in Section 4.3 following Corollary 4 , and we therefore omit it here for reasons of space. 